\title{
Synthesis of Biobased Phloretin Analogues: An Access to Antioxidant and Anti-Tyrosinase Compounds for Cosmetic Applications
}

\author{
Laurène Minsat ${ }^{1,2} \mathbb{D}$, Cédric Peyrot ${ }^{1, * \mathbb{D}}$, Fanny Brunissen ${ }^{1} \mathbb{D}$, Jean-Hugues Renault ${ }^{2}$ and Florent Allais ${ }^{1, * \mathbb{D}}$ \\ 1 URD Agro-Biotechnologies Industrielles (ABI), CEBB (Centre Européen de Biotechnologie et de \\ Bioéconomie), AgroParisTech, 51110 Pomacle, France; laurene.minsat@agroparistech.fr (L.M.); \\ fanny.brunissen@agroparistech.fr (F.B.) \\ 2 Université de Reims Champagne-Ardenne, CNRS, ICMR 7312, 51097 Reims, France; \\ Jean-hugues.renault@univ-reims.fr \\ * Correspondence: cedric.peyrot@agroparistech.fr (C.P.); florent.allais@agroparistech.fr (F.A.)
}

check for updates

Citation: Minsat, L.; Peyrot, C.; Brunissen, F.; Renault, J.-H.; Allais, F. Synthesis of Biobased Phloretin Analogues: An Access to Antioxidant and Anti-Tyrosinase Compounds for Cosmetic Applications. Antioxidants 2021, 10, 512. https://doi.org/ 10.3390/antiox10040512

Academic Editors: Maria Modica and Mohammad Hossain

Received: 25 January 2021

Accepted: 22 March 2021

Published: 25 March 2021

Publisher's Note: MDPI stays neutral with regard to jurisdictional claims in published maps and institutional affiliations.

Copyright: (c) 2021 by the authors. Licensee MDPI, Basel, Switzerland. This article is an open access article distributed under the terms and conditions of the Creative Commons Attribution (CC BY) license (https:/ / creativecommons.org/licenses/by/ $4.0 /)$.

\begin{abstract}
The current cosmetic and nutraceutical markets are characterized by a strong consumer demand for a return to natural products that are less harmful to both the consumers and the environment than current petrosourced products. Phloretin, a natural dihydrochalcone (DHC) found in apple, has been widely studied for many years and identified as a strong antioxidant and antityrosinase ingredient for cosmetic formulations. Its low concentration in apples does not allow it to be obtained by direct extraction from biomass in large quantities to meet market volumes and prices. Moreover, its remarkable structure prevents its synthesis through a green process. To overcome these issues, the synthesis of phloretin analogs appears as an alternative to access valuable compounds that are potentially more active than phloretin itself. Under such considerations, 12 chalcones (CHs) and 12 dihydrochalcones (DHCs) were synthesized through a green Claisen-Schmidt condensation using bio-based reagents. In order to evaluate the potential of these molecules, radical scavenging DPPH and anti-tyrosinase tests have been conducted. Moreover, the UV filtering properties and the stability of these analogs towards UV-radiations have been evaluated. Some molecules showed competitive antioxidant and anti-tyrosinase activities regarding phloretin. Two compounds in particular showed $\mathrm{EC}_{50}$ lower than phloretin, one chalcone and one dihydrochalcone.
\end{abstract}

Keywords: phloretin analogues; chalcones; dihydrochalcones; Claisen-Schmidt condensation; DPPH test; anti-tyrosinase; antioxidant

\section{Introduction}

In the past ten years, special attention has been paid to phloretin, a naturally occurring flavonoid found in apple especially in peels [1] but more consistently in leaves [2], branches, barks and roots [3]. It can be recovered from processed apple products and by-products, such as apple pomace resulting from cider production [4]. Phloretin is a dihydrochalcone (DHC) composed of two aromatic rings, commonly named A and B, bearing hydroxy groups in positions $2^{\prime}, 4,4^{\prime}$ and $6^{\prime}$ connected by a three-carbon $\alpha, \beta$-unsaturated carbonyl system (Figure 1). This remarkable structure provides phloretin with many interesting biological activities that have been widely studied [5-11]. For instance, Barreca et al. demonstrated strong antimicrobial activities for phloretin in particular against Gram-positive bacteria such as Staphylococcus aureus and Listeria monocytogenes. They also highlighted the importance of the free hydroxy moiety in position $2^{\prime}$ and the absence of glycosyl moiety (compared to phlorizin, the best-known phloretin glycoside) [12]. Antimicrobial properties are widely sought in view of the many criticisms against the main preservatives used in cosmetics, such as parabens and phenoxyethanol [13]. In addition, phloretin inhibits the formation of some bacterial biofilms, which is a serious human health issue due to these 
bacteria's increasing resistance to antimicrobial treatments and their ubiquity in medical and engineering environments [14,15].

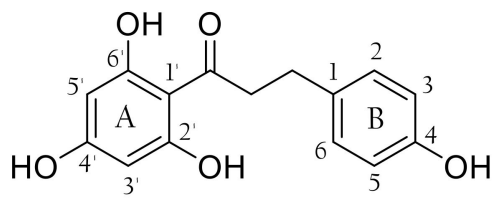

Phloretin

Figure 1. Structure of phloretin.

Several studies also reported strong antioxidant activities, such as that of Rezk et al., who linked it to the presence of the three hydroxyls groups on the A-ring and the carbonyl group [16]. In fact, phloretin is able to (1) scavenge hydroxyl radicals, stable free radicals such as 2,2-diphenyl-1-picrylhydrazyl (DPPH), peroxynitrite, and (2) prevent lipid peroxidation $[16,17]$.

Additionally, as skin cancers are increasing considerably, linked to overexposure to the sun $[18,19]$, phloretin acts also as a potential inhibitor of tyrosinase, the key enzyme in skin pigment disorders. Indeed, phloretin showed a significant inhibition of the activity of mushroom tyrosinase in vitro and tyrosinase from human melanocytes [20,21]. Therefore, it can be used as a topical lightening agent in cosmetics to treat skin pigmentary disorder. Recently, a study led by L'Oreal USA ${ }^{\circledR}$ showed the UV protection for human skin provided by phloretin formulated with vitamin $C$ and ferulic acid. Furthermore, it stabilizes the formulation and increases the skin availability of the other components [22]. Indeed, phloretin can be used as a skin penetration enhancer in formulations thanks to its ability to interact with bilayer membranes, increasing their fluidity [11,23].

In this context, it is not surprising to find phloretin as an active ingredient in cosmetic formulations. However, the small amount of this compound present in the biomass pushes us to design chemical synthestic pathways, allowing us to meet the potential increase in demand. Therefore, it appears interesting to explore its chemical synthesis or hemisynthesis through a green process to fulfill the current requirements of targeted markets. Nevertheless, only a few studies have reported such synthetic approaches. Siddaiah et al. proposed Friedel-Crafts acylation pathway between phenols and dihydrocinnamic acids using borontrifluoride etherate $\left(\mathrm{BF}_{3} \cdot \mathrm{Et}_{2} \mathrm{O}\right)$, but phloretin was isolated in poor yields $(30 \%)$ [24]. Another pathway was proposed, requiring protection steps for the phloretin derivatives synthesis. It used benzyl groups to prevent the intramolecular cyclization due to the hydroxyl group in the ortho position of phloretin precursor, the naringenin chalcone [25]. Indeed, this hydroxyl constitutes a real limitation as it readily leads to a Michael addition to form naringenin (Scheme 1).

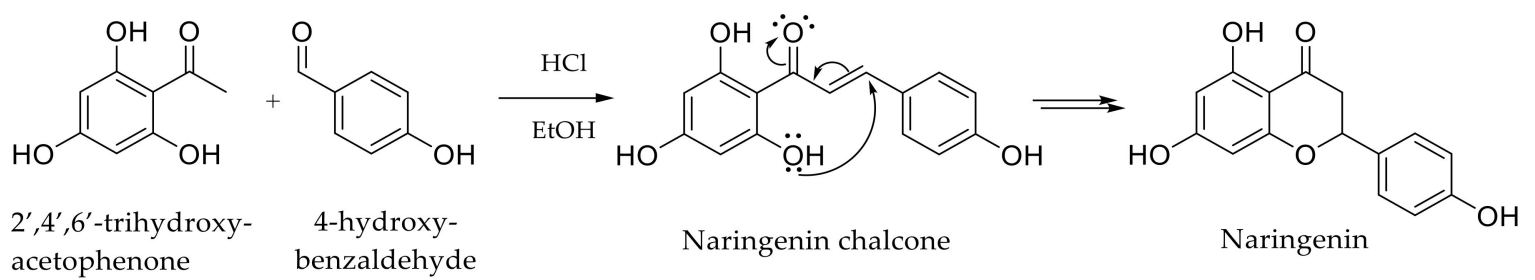

Scheme 1. Intramolecular cyclization occurring during phloretin synthesis through a Claisen-Schmidt condensation catalyzed by $\mathrm{HCl}$.

To overcome these issues, the synthesis of phloretin analogs appears as an alternative to access valuable compounds potentially more active than phloretin itself. The easiest pathway to access DHCs consists of (1) synthesizing the corresponding chalcone, and (2) reducing it in presence of hydrogen with palladium on carbon [26]. Chalcones (CHs) are $\alpha, \beta$-unsaturated analogs of DHCs, naturally occurring in various plants and widely studied 
for many years for their biological properties $[27,28]$. While the reduction step (2) is rather classic, there are many ways to synthesize CHs (1). Claisen-Schmidt condensation between substituted or unsubstituted acetophenone and benzaldehyde is the most frequently used method $[27,29,30]$. This reaction can be performed in acidic or basic conditions in polar solvents. In general, basic reagents are preferred, mainly $\mathrm{NaOH}$ [31], $\mathrm{KOH}$ [26], $\mathrm{NaH}$ [32], as they often lead to high yields (40-80\%) while acidic reagents, such as $\mathrm{HCl}$ or boric acid, lead to a wider range of yields from $10 \%$ to $80 \%$ [28,33,34]. However, the use of an acid catalyst allows side reactions to be prevented, such as the Cannizzaro reaction, which occurs in strongly alkaline conditions [35]. Recently, greener conditions were developed using reusable catalysts such as Amberlyst-15 and Amberlite-200C, which are commercial acidresins, or even solid base catalyst (e.g., poly( $N$-vinylimidazole)) coupled with ultrasonic irradiation under solvent-free conditions [36,37]. Although Claisen-Schmidt condensation is the most widely used method due to its simple procedure, other approaches have been described. Different coupling reactions, for instance, Sonogashira [38], Heck [39] or Suzuki couplings [40], were employed as novel strategies for molecule synthesis including chalcones. Xu et al. described an innovative microwave-assisted Wittig reaction leading to excellent yields, but only in small quantities and not quite sustainable for the industry [41].

The purpose of this work is to first synthesize a library of 24 phloretin analogsincluding seven new compounds-with higher potential than phloretin itself by modulating the substituents on A and B rings to tune the properties for a potential valorization in the cosmetic or nutraceutical sector. In order to limit the use of petrosourced precursors, metal catalysts and multi-step synthesis, and to respect the green chemistry principles, we aimed at synthetizing phloretin analogs through a simple two-step process: an $\mathrm{HCl}$ catalyzed Claisen-Schmidt condensation using bio-based reagents, followed by a catalytic palladium-catalyzed hydrogenation. This method not only offers various advantages such as simple and quick procedures and high reaction yields, but also avoids side reactions or protection steps. Moreover, the use of $\mathrm{HCl}$ in catalytic amount significantly limits the environmental impact of this approach. The antioxidant properties, the anti-tyrosinase activity and the anti-UV activity of the 24 analogs were then assessed to establish Structure-Activity Relationships. To the best of our knowledge, this is the first time that the anti-tyrosinase and anti-UV properties of phloretin analogs have been determined and discussed.

\section{Materials and Methods}

\subsection{Materials}

All reagents and solvents were purchased from Sigma Aldrich (St. Louis, MO, USA), TCI (Tokyo, Japan) or Fisher Scientific (Hampton, NH, USA). All chemicals were used directly without purification.

\subsection{Purification and Characterization}

The $\mathrm{CH}$ and DHC purifications were performed by flash chromatography on a flashprep PuriFlash ${ }^{\circledR} 4100$ LC system from Interchim (Montluçon, France) with prepacked silica columns ( $30 \mu \mathrm{m}$, Interchim PF-Si30-HP) and dual-wavelength collection $(\lambda=254$ and $280 \mathrm{~nm})$. Mixtures of cyclohexane and ethyl acetate were used as eluant.

The CHs and DHCs characterizations were realized by NMR and mass spectrometry. ${ }^{1} \mathrm{H}-\mathrm{NMR}$ spectra were recorded on a Brucker Fourier 300 (300 MHz) (Billerica, MA, USA). Residual Acetone- $d_{6}$ and DMSO- $d_{6}$ protons signals at $\delta 2.05$ and $2.5 \mathrm{ppm}$, respectively, were used for the calibration. Data are reported as follows: chemical shift ( $\delta \mathrm{ppm})$, multiplicity ( $\mathrm{s}=$ singlet, $\mathrm{d}=$ doublet, $\mathrm{t}=$ triplet, $\mathrm{dd}=$ doublet of doublets, and $\mathrm{m}=$ multiplet), coupling constant $(\mathrm{Hz})$, integration and assignment. ${ }^{13} \mathrm{C}-\mathrm{NMR}$ spectra were recorded on a Bruker Fourier $300(75 \mathrm{MHz})$ (Billerica, MA, USA). Residual Acetone- $d_{6}$ and DMSO- $d_{6}$ protons signals at $\delta 29.84 \mathrm{ppm}$ and $39.52 \mathrm{ppm}$, respectively, were used for the calibration. Data are reported as follows: chemical shift $(\delta \mathrm{ppm})$, assignment. All NMR assignments were made using COSY, HMBC and HSQC spectra. 1D and 2D NMR spectra $\left({ }^{1} \mathrm{H},{ }^{13} \mathrm{C}, \mathrm{HSQC}, \mathrm{HMBC}\right.$, and COSY) were recorded using standard Bruker pulse programs. 
High-resolution mass spectrometry was performed on an Agilent, 1290 system, equipped with a PDA UV detector and a 6545 Q-TOF mass spectrometer (Wilmington, DE, USA). The source was equipped with a JetStream ESI probe operating at atmospheric pressure. The electrospray interface operated with the following parameters: scanning range of 50-1000, nebulizer 35 psi, gas temperature $325^{\circ} \mathrm{C}$, gas flow $8 \mathrm{~L} / \mathrm{min}$, sheath da temperature $350{ }^{\circ} \mathrm{C}$, sheath gas flow $11 \mathrm{~L} / \mathrm{min}$. Elution was performed using a Zorbax Eclipse plus C18 $\left(1.8 \mu \mathrm{m}, 50 \times 2.1 \mathrm{~mm}^{2}\right.$; Agilent), with the following mobile phases: $0.1 \%$ formic acid in water (solvent A) and acetonitrile (solvent B). The flow rate was set at $0.4 \mathrm{~mL} / \mathrm{min}$ and according to the gradient $0-3 \mathrm{~min}$ at $5 \%$ of $\mathrm{B}, 3-4 \mathrm{~min}$ from $5 \%$ to $10 \%$ B, 4-13 min from $10 \%$ to $99 \%$ B, 13-16 min at $99 \%$ B, 16-18 min from $99 \%$ to $5 \% \mathrm{~B}$. The column was heated at $40{ }^{\circ} \mathrm{C}$ and the sample injection volume was $1 \mu \mathrm{L}$.

FTIR spectra were recorded on a Cary 630 FTIR Spectrometer by Agilent (Wilmington, DE, USA).

Melting points were recorded on a Mettler Toledo MP50 Melting Point System (Greifensee, Switzerland) (heating at $3^{\circ} \mathrm{C} / \mathrm{min}$ ).

\subsection{General Procedure for Chalcones Synthesis}

All CHs were synthesized by a Claisen-Schmidt condensation using EtOH as solvent and $\mathrm{HCl}$ as catalyst. The corresponding substituted acetophenone (1.0 equiv) and benzaldehyde (1.2 equiv) were dissolved in ethanol in a round-bottom flask topped by an air cooling column. A few drops of $\mathrm{HCl}$ (37\%) were added, and the reaction mixture was refluxed overnight with continued stirring. The solvent was evaporated under reduced pressure, and the product was dry loaded onto Celite ${ }^{\circledR}$ to be purified through flash chromatography on silica gel.

For each compound, the numbers for the NMR assignment are related to the labeling in the NMR spectra in the Supplementary Materials.

4,4'-dihydroxy-chalcone (a1): purification (cyclohexane/ethyl acetate: 50/50) led to a yellow powder $(76 \%)$. m.p. $202-203^{\circ} \mathrm{C}, \mathrm{UV}: \lambda_{\max }(\mathrm{EtOH}, \mathrm{nm}) 350, \varepsilon\left(\mathrm{L} \cdot \mathrm{mol}^{-1} \cdot \mathrm{cm}^{-1}\right) 87,739$. FTIR (pure) $v\left(\mathrm{~cm}^{-1}\right): 3465,3428,1639,1586 .{ }^{1} \mathrm{H}-\mathrm{NMR}\left(300 \mathrm{MHz}\right.$, Acetone- $\left.d_{6}\right) \delta: 8.07$ $\left(\mathrm{d}, J=8.8 \mathrm{~Hz}, 2 \mathrm{H}, \mathrm{H} 9-9^{\prime}\right), 7.70\left(\mathrm{~d}, J=8.6 \mathrm{~Hz}, 2 \mathrm{H}, \mathrm{H} 5-5^{\prime}\right), 7.69(\mathrm{~s}, 2 \mathrm{H}, \mathrm{H} 2$ and H3), $6.96(\mathrm{~d}$, $\left.J=8.6 \mathrm{~Hz}, 2 \mathrm{H}, \mathrm{H} 10-10^{\prime}\right), 6.91\left(\mathrm{~d}, J=8.7 \mathrm{~Hz}, 2 \mathrm{H}, \mathrm{H6}-6^{\prime}\right) .{ }^{13} \mathrm{C}-\mathrm{NMR}\left(75 \mathrm{MHz}\right.$, Acetone- $\left.d_{6}\right)$

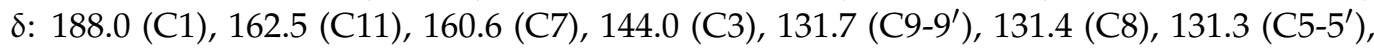
127.9 (C4), $119.7(\mathrm{C} 2), 116.7\left(\mathrm{C}^{2}-6^{\prime}\right), 116.1\left(\mathrm{C} 10-10^{\prime}\right)$. HRMS: $m / z[\mathrm{M}+\mathrm{H}]^{+}$calculated for $\mathrm{C}_{15} \mathrm{H}_{13} \mathrm{O}_{3}: 241.0864$ found: 241.0864 .

3,4,4'-trihydroxy-chalcone (a2): purification (cyclohexane/ethyl acetate: 50/50) led to a yellow powder $(80 \%)$. m.p. $189-190^{\circ} \mathrm{C}, \mathrm{UV}: \lambda_{\max }(\mathrm{EtOH}, \mathrm{nm}) 365, \varepsilon\left(\mathrm{L} \cdot \mathrm{mol}^{-1} \cdot \mathrm{cm}^{-1}\right) 29,139$. FTIR (pure) $v\left(\mathrm{~cm}^{-1}\right): 3476-3191,1644,1589 .{ }^{1} \mathrm{H}-\mathrm{NMR}\left(300 \mathrm{MHz}, \mathrm{DMSO}-d_{6}\right) \delta: 8.01(\mathrm{~d}$, $\left.J=8.7 \mathrm{~Hz}, 2 \mathrm{H}, \mathrm{H} 11-11^{\prime}\right), 7.59(\mathrm{~d}, J=15.5 \mathrm{~Hz}, 1 \mathrm{H}, \mathrm{H} 3), 7.52(\mathrm{~d}, J=15.4 \mathrm{~Hz}, 1 \mathrm{H}, \mathrm{H} 2), 7.22(\mathrm{~d}$, $J=1.6 \mathrm{~Hz}, 1 \mathrm{H}, \mathrm{H} 5), 7.15(\mathrm{dd}, J=1.7 \mathrm{~Hz}, 8.2 \mathrm{~Hz}, 1 \mathrm{H}, \mathrm{H9}), 6.87$ (d, $\left.J=8.6 \mathrm{~Hz}, 2 \mathrm{H}, \mathrm{H} 12-12^{\prime}\right)$, $6.79(\mathrm{~d}, J=8.2 \mathrm{~Hz}, 1 \mathrm{H}, \mathrm{H} 8) .{ }^{13} \mathrm{C}-\mathrm{NMR}\left(75 \mathrm{MHz}, \mathrm{DMSO}-d_{6}\right) \delta: 187.1$ (C1), $161.9(\mathrm{C} 13)$, 148.4 (C7), 145.6 (C6), 143.7 (C3), 130.9 (C11-11'), 129.5 (C10), 126.5 (C4), 121.9 (C9), 118.4 (C2), 115.8 (C8), 115.4 (C5), $115.3\left(\mathrm{C} 12-12^{\prime}\right)$. HRMS: $m / z[\mathrm{M}+\mathrm{H}]^{+}$calculated for $\mathrm{C}_{15} \mathrm{H}_{13} \mathrm{O}_{4}$ : 257.0814 found: 257.0815 .

$3^{\prime}, 4,4^{\prime}$-trihydroxy-chalcone (a3): purification (cyclohexane/ethyl acetate: 50/50) led to a yellow powder $(68 \%)$. m.p. $176-177^{\circ} \mathrm{C}, \mathrm{UV}: \lambda_{\max }(\mathrm{EtOH}, \mathrm{nm}) 358, \varepsilon\left(\mathrm{L} \cdot \mathrm{mol}^{-1} \cdot \mathrm{cm}^{-1}\right) 30,628$. FTIR (pure) $v\left(\mathrm{~cm}^{-1}\right): 3425-3198,1631,1597 .{ }^{1} \mathrm{H}-\mathrm{NMR}\left(300 \mathrm{MHz}, \mathrm{DMSO}-d_{6}\right) \delta: 7.68(\mathrm{~d}$, $\left.J=8.6 \mathrm{~Hz}, 2 \mathrm{H}, \mathrm{H} 5-5^{\prime}\right), 7.58(\mathrm{~m}, 3 \mathrm{H}, \mathrm{H} 2, \mathrm{H} 3$ and $\mathrm{H} 9), 7.49(\mathrm{~d}, J=1.8 \mathrm{~Hz}, 1 \mathrm{H}, \mathrm{H} 13), 6.85(\mathrm{~d}, J$ $=7.9 \mathrm{~Hz}, 1 \mathrm{H}, \mathrm{H10}), 6.82\left(\mathrm{~d}, J=8.0 \mathrm{~Hz}, 2 \mathrm{H}, \mathrm{H6}-6^{\prime}\right) .{ }^{13} \mathrm{C}-\mathrm{NMR}\left(75 \mathrm{MHz}, \mathrm{DMSO}-d_{6}\right) \delta: 187.1$ (C1), 159.8 (C7), 150.6 (C11), 145.4 (C12), 142.9 (C3), 130.7 (C5-5'), 129.9 (C8), 126.0 (C4), 121.8 (C9), 118.6 (C2), 115.8 (C6-6'), 115.3 (C10 or C13), 115.1 (C10 or C13). HRMS: $m / z$ $[\mathrm{M}+\mathrm{H}]^{+}$calculated for $\mathrm{C}_{15} \mathrm{H}_{13} \mathrm{O}_{4}: 257.0814$ found: 257.0815 .

3,3', 4,4'-tetrahydroxy-chalcone (a4): purification (cyclohexane/ethyl acetate: 45/55) led to a yellow powder $(62 \%)$. m.p. $209-210{ }^{\circ} \mathrm{C}, \mathrm{UV}: \lambda_{\max }(\mathrm{EtOH}, \mathrm{nm}) 371, \varepsilon\left(\mathrm{L} \cdot \mathrm{mol}^{-1} \cdot \mathrm{cm}^{-1}\right)$ 
47,295. FTIR (pure) $v\left(\mathrm{~cm}^{-1}\right): 3472,3267,1635,1592 .{ }^{1} \mathrm{H}-\mathrm{NMR}\left(300 \mathrm{MHz}, \mathrm{DMSO}-d_{6}\right) \delta: 7.56$ (dd, $J=1.7 \mathrm{~Hz}, 8.25 \mathrm{~Hz}, 1 \mathrm{H}, \mathrm{H} 11), 7.50$ (s, 2H, H2 and H3), 7.47 (d, J = 1.7 Hz, 1H, H15), 7.20 (s, 1H, H5), 7.13 (dd, J = 1.4 Hz, 8.2 Hz, 1H, H9), 6.84 (d, J = 8.2 Hz, 1H, H12), 6.79 (d, $J=8.1 \mathrm{~Hz}, 1 \mathrm{H}, \mathrm{H} 8) .{ }^{13} \mathrm{C}-\mathrm{NMR}\left(75 \mathrm{MHz}, \mathrm{DMSO}-d_{6}\right) \delta: 187.1$ (C1), 150.6 (C13), 148.4 (C7), 145.6 (C6 or C14), 145.5 (C6 or C14), 143.4 (C3), 130.0 (C10), 126.5 (C4), 121.8 (C9 and C11), 118.5 (C2), 115.8 (C8), 115.3 (C5 and C15), 115.1 (C12). HRMS: $m / z[\mathrm{M}+\mathrm{H}]^{+}$calculated for $\mathrm{C}_{15} \mathrm{H}_{13} \mathrm{O}_{5}: 273.0763$ found: 273.0762 .

4,4'-dihydroxy-3-methoxy-chalcone (a5): purification (cyclohexane/ethyl acetate: 50/50) led to a yellow powder $(80 \%)$. m.p. $224-227^{\circ} \mathrm{C}, \mathrm{UV}: \lambda_{\max }(\mathrm{EtOH}, \mathrm{nm}) 362, \varepsilon\left(\mathrm{L} \cdot \mathrm{mol}^{-1} \cdot \mathrm{cm}^{-1}\right)$ 33,032. FTIR (pure) $v\left(\mathrm{~cm}^{-1}\right): 3395,1638,1587 .{ }^{1} \mathrm{H}-\mathrm{NMR}\left(300 \mathrm{MHz}, \mathrm{DMSO}-\mathrm{d}_{6}\right) \delta: 8.06(\mathrm{~d}$, $\left.J=8.7 \mathrm{~Hz}, 2 \mathrm{H}, \mathrm{H} 11-11^{\prime}\right), 7.73(\mathrm{~d}, J=15.5 \mathrm{~Hz}, 1 \mathrm{H}, \mathrm{H} 2), 7.60(\mathrm{~d}, J=15.5 \mathrm{~Hz}, 1 \mathrm{H}, \mathrm{H} 3), 7.49(\mathrm{~d}$, $J=1.6 \mathrm{~Hz}, 1 \mathrm{H}, \mathrm{H} 5), 7.24(\mathrm{dd}, J=1.7 \mathrm{~Hz}, 8.2 \mathrm{~Hz}, 1 \mathrm{H}, \mathrm{H9}), 6.89$ (d, $\left.J=8.7 \mathrm{~Hz}, 2 \mathrm{H}, \mathrm{H} 12-12^{\prime}\right)$, $6.82(\mathrm{~d}, J=8.1 \mathrm{~Hz}, 1 \mathrm{H}, \mathrm{H} 8), 3.87$ (s, 3H, H14). ${ }^{13} \mathrm{C}-\mathrm{NMR}\left(75 \mathrm{MHz}, \mathrm{DMSO}-d_{6}\right) \delta: 187.0(\mathrm{C} 1)$, 162.0 (C13), 149.4 (C7), 148.0 (C6), 143.7 (C3), 131.0 (C11-11' ), 129.4 (C10), 126.5 (C4), 123.9 (C9), 118.7 (C2), 115.6 (C8), 115.3 (C12-12'), 111.5 (C5), 55.8 (C14). HRMS: $m / z$ [M+H] ${ }^{+}$ calculated for $\mathrm{C}_{16} \mathrm{H}_{15} \mathrm{O}_{4}$ : 271.0970 found: 271.0973 .

4, $\mathbf{4}^{\prime}$-dihydroxy-3'-methoxy-chalcone (a6): purification (cyclohexane/ethyl acetate: 45/55) led to a yellow powder (78\%). m.p. $187-188^{\circ} \mathrm{C}, \mathrm{UV}: \lambda_{\max }(\mathrm{EtOH}, \mathrm{nm}) 360, \varepsilon\left(\mathrm{L} \cdot \mathrm{mol}^{-1} \cdot \mathrm{cm}^{-1}\right)$ 29,982. FTIR (pure) $v\left(\mathrm{~cm}^{-1}\right): 3383,3211,1645,1587 .{ }^{1} \mathrm{H}-\mathrm{NMR}\left(300 \mathrm{MHz}\right.$, Acetone- $\left.d_{6}\right) \delta$ : $7.78(\mathrm{dd}, J=2.0 \mathrm{~Hz}, 8.3 \mathrm{~Hz}, 1 \mathrm{H}, \mathrm{H} 9), 7.69\left(\mathrm{~m}, 5 \mathrm{H}, \mathrm{H} 2, \mathrm{H} 3, \mathrm{H} 5-5^{\prime}\right.$ and H13), $6.95(\mathrm{~d}, J=8.3 \mathrm{~Hz}$, 1H, H10), 6.91 (d, $J=8.7 \mathrm{~Hz}, 2 \mathrm{H}, \mathrm{H6}-6$ ') 3.94 (s, 3H, H14). ${ }^{13} \mathrm{C}-\mathrm{NMR}\left(75 \mathrm{MHz}\right.$, Acetone- $\left.d_{6}\right)$

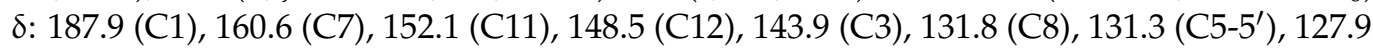
(C4), 124.1 (C9), 119.6 (C2), 116.7 (C6-6'), 115.4 (C10), 112.0 (C13), 56.3 (C14). HRMS: $\mathrm{m} / z$ $[\mathrm{M}+\mathrm{H}]^{+}$calculated for $\mathrm{C}_{16} \mathrm{H}_{15} \mathrm{O}_{4}: 271.0970$ found: 271.0972 .

3,4,4'-trihydroxy-3-methoxy-chalcone (a7): purification (cyclohexane/ethyl acetate: 45/55) led to a yellow powder (72\%). m.p. $172-173^{\circ} \mathrm{C}, \mathrm{UV}: \lambda_{\max }(\mathrm{EtOH}, \mathrm{nm}) 372, \varepsilon\left(\mathrm{L} \cdot \mathrm{mol}^{-1} \cdot \mathrm{cm}^{-1}\right)$ 37,725. FTIR (pure) $v\left(\mathrm{~cm}^{-1}\right): 3450,3125,1640,1588 .{ }^{1} \mathrm{H}-\mathrm{NMR}\left(300 \mathrm{MHz}\right.$, Acetone- $\left.d_{6}\right) \delta$ : $7.77(\mathrm{dd}, J=2.0 \mathrm{~Hz}, 8.28 \mathrm{~Hz}, 1 \mathrm{H}, \mathrm{H} 11), 7.69$ (d, $J=1.9 \mathrm{~Hz}, 1 \mathrm{H}, \mathrm{H} 15), 7.64(\mathrm{~s}, 2 \mathrm{H}, \mathrm{H} 2$ and H3), 7.31 (d, J = 2.1 Hz, 1H, H5), $7.18(\mathrm{dd}, J=2.1 \mathrm{~Hz}, 8.19 \mathrm{~Hz}, 1 \mathrm{H}, \mathrm{H} 9), 6.95$ (d, J = 8.3 Hz, $1 \mathrm{H}, \mathrm{H} 12), 6.89$ (d, $J=8.2 \mathrm{~Hz}, 1 \mathrm{H}, \mathrm{H} 8), 3.94$ (s, 3H, H16). ${ }^{13} \mathrm{C}-\mathrm{NMR}\left(75 \mathrm{MHz}\right.$, Acetone- $\left.d_{6}\right)$

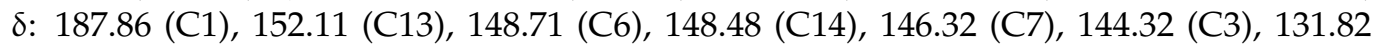
(C10), 128.55 (C4), 124.09 (C11), 122.97 (C9), 119.71 (C2), 116.36 (C8), 115.66 (C5), 115.42 (C12), 112.02 (C15), 56.31 (C16). HRMS: $m / z[\mathrm{M}+\mathrm{H}]^{+}$calculated for $\mathrm{C}_{16} \mathrm{H}_{15} \mathrm{O}_{5}: 287.0919$ found: 287.0917.

$3^{\prime}, 4,4^{\prime}$-trihydroxy-3-methoxy-chalcone (a8): purification (cyclohexane/ethyl acetate: 45/55) led to a yellow powder $(78 \%)$. Degradation temperature $186-187{ }^{\circ} \mathrm{C}$, UV: $\lambda_{\max }(\mathrm{EtOH}$, $\mathrm{nm}) 365, \varepsilon\left(\mathrm{L} \cdot \mathrm{mol}^{-1} \cdot \mathrm{cm}^{-1}\right)$ 31,067. FTIR (pure) $v\left(\mathrm{~cm}^{-1}\right): 3517-3234,1647,1591 .{ }^{1} \mathrm{H}-\mathrm{NMR}$ $\left(300 \mathrm{MHz}, \mathrm{DMSO}-d_{6}\right) \delta: 7.68(\mathrm{~d}, J=15.5 \mathrm{~Hz}, 1 \mathrm{H}, \mathrm{H} 2), 7.63(\mathrm{dd}, J=2.1 \mathrm{~Hz}, 8.4 \mathrm{~Hz}, 1 \mathrm{H}, \mathrm{H} 11)$, $7.57(\mathrm{~d}, J=15.4 \mathrm{~Hz}, 1 \mathrm{H}, \mathrm{H} 3), 7.5(\mathrm{~d}, J=2.1 \mathrm{~Hz}, 1 \mathrm{H}, \mathrm{H} 15), 7.47(\mathrm{~d}, J=1.8 \mathrm{~Hz}, 1 \mathrm{H}, \mathrm{H} 5), 7.22$ $(\mathrm{dd}, J=1.8 \mathrm{~Hz}, 8.3 \mathrm{~Hz}, 1 \mathrm{H}, \mathrm{H9}), 6.85(\mathrm{~d}, J=8.3 \mathrm{~Hz}, 1 \mathrm{H}, \mathrm{H} 12), 6.81(\mathrm{~d}, J=8.1 \mathrm{~Hz}, 1 \mathrm{H}, \mathrm{H} 8)$, 3.86 (s, 3H, H16). ${ }^{13} \mathrm{C}-\mathrm{NMR}\left(75 \mathrm{MHz}, \mathrm{DMSO}-d_{6}\right) \delta: 187.1$ (C1), 150.6 (C13), 149.3 (C7), 148.0 (C6), 145.4 (C14), 143.3 (C3), 130.0 (C10), 126.5 (C4), 123.8 (C9), 121.9 (C11), 118.8 (C2), 115.6 (C8), 115.4 (C15), 115.0 (C12), 111.4 (C5), 55.8 (C16). HRMS: $m / z[\mathrm{M}+\mathrm{H}]^{+}$calculated for $\mathrm{C}_{16} \mathrm{H}_{15} \mathrm{O}_{5}$ : 287.0919 found: 287.0922.

4,4'-dihydroxy-3',5'-dimethoxy-chalcone (a9): purification (cyclohexane/ethyl acetate: $50 / 50)$ led to a yellow powder (65\%). m.p. 170-171 ${ }^{\circ} \mathrm{C}, \mathrm{UV}: \lambda_{\max }(\mathrm{EtOH}, \mathrm{nm}) 363, \varepsilon$ $\left(\mathrm{L} \cdot \mathrm{mol}^{-1} \cdot \mathrm{cm}^{-1}\right)$ 27,390. FTIR (pure) $v\left(\mathrm{~cm}^{-1}\right): 3508,1637,1591 .{ }^{1} \mathrm{H}-\mathrm{NMR}(300 \mathrm{MHz}$, Acetone- $\left.d_{6}\right) \delta: 7.73\left(\mathrm{~s}, 2 \mathrm{H}, \mathrm{H} 2\right.$ and H3), $7.69\left(\mathrm{~d}, J=8.6 \mathrm{~Hz}, 2 \mathrm{H}, \mathrm{H} 5-5^{\prime}\right), 7.49\left(\mathrm{~s}, 2 \mathrm{H}, \mathrm{H} 9-9^{\prime}\right)$, $6.91\left(\mathrm{~d}, J=8.6 \mathrm{~Hz}, 2 \mathrm{H}, \mathrm{H6}-6^{\prime}\right), 3.93\left(\mathrm{~s}, 6 \mathrm{H}, \mathrm{H} 12-12^{\prime}\right) .{ }^{13} \mathrm{C}-\mathrm{NMR}\left(75 \mathrm{MHz}\right.$, Acetone- $\left.d_{6}\right) \delta$ : 187.9 (C1), 160.6 (C7), 148.6 (C10-10'), 144.1 (C3), 141.8 (C11), 131.4 (C5-5'), 130.3 (C8), 127.9 (C4), $119.6(\mathrm{C} 2), 116.7\left(\mathrm{C}^{2}-6^{\prime}\right), 107.3\left(\mathrm{C}^{\prime}-9^{\prime}\right), 56.8\left(\mathrm{C} 12-12^{\prime}\right)$. HRMS: $m / z[\mathrm{M}+\mathrm{H}]^{+}$calculated for $\mathrm{C}_{17} \mathrm{H}_{17} \mathrm{O}_{5}$ : 301.1076 found: 301.1078 . 
4, $\mathbf{4}^{\prime}$-dihydroxy-3,3'-dimethoxy-chalcone (a10): purification (cyclohexane/ethyl acetate: $50 / 50)$ led to a yellow powder (69\%). m.p. $126-127^{\circ} \mathrm{C}$, UV: $\lambda_{\max }(\mathrm{EtOH}, \mathrm{nm}) 369, \varepsilon$ $\left(\mathrm{L} \cdot \mathrm{mol}^{-1} \cdot \mathrm{cm}^{-1}\right)$ 29,411. FTIR (pure) $v\left(\mathrm{~cm}^{-1}\right): 3368,1641,1589 .{ }^{1} \mathrm{H}-\mathrm{NMR}(300 \mathrm{MHz}$, Acetone- $\left.d_{6}\right) \delta: 7.70(\mathrm{dd}, J=2.0 \mathrm{~Hz}, 8.3 \mathrm{~Hz}, 1 \mathrm{H}, \mathrm{H} 11), 7.72(\mathrm{~d}, J=3.5 \mathrm{~Hz}, 2 \mathrm{H}, \mathrm{H} 2$ and H3), $7.68(\mathrm{~d}, J=2.0 \mathrm{~Hz}, 1 \mathrm{H}, \mathrm{H} 15), 7.48(\mathrm{~d}, J=2.0 \mathrm{~Hz}, 1 \mathrm{H}, \mathrm{H} 5), 7.30(\mathrm{dd}, J=2.0 \mathrm{~Hz}, 8.2 \mathrm{~Hz}, 1 \mathrm{H}$, H9), $6.94(\mathrm{~d}, J=8.3 \mathrm{~Hz}, 1 \mathrm{H}, \mathrm{H} 12), 6.89$ (d, $J=8.2 \mathrm{~Hz}, 1 \mathrm{H}, \mathrm{H} 8), 3.94$ (s, 3H, H16 or H17), 3.93 (s, 3H, H16 or H17). ${ }^{13} \mathrm{C}-\mathrm{NMR}$ (75 MHz, Acetone- $d_{6}$ ) $\delta: 187.8$ (C1), 152.1 (C13), 150.1 (C7), 148.7 (C6), 148.5 (C14), 144.4 (C3), 131.8 (C10), 128.3 (C4), 124.2 (C9), 124.1 (C11), 119.8 (C2), 116.1 (C8), 115.3 (C12), 112 (C15), 111.9 (C5), 56.4 (C16 or C17), 56.3 (C16 or C17). HRMS: $m / z[\mathrm{M}+\mathrm{H}]^{+}$calculated for $\mathrm{C}_{17} \mathrm{H}_{17} \mathrm{O}_{5}: 301.1076$ found: 301.1077 .

4,4'-dihydroxy-3,3',5-trimethoxy-chalcone (a11): purification (cyclohexane/ethyl acetate: $50 / 50)$ led to a yellow powder $(47 \%)$. m.p. $159-160{ }^{\circ} \mathrm{C}$, UV: $\lambda_{\max }(\mathrm{EtOH}, \mathrm{nm}) 375, \varepsilon$ $\left(\mathrm{L} \cdot \mathrm{mol}^{-1} \cdot \mathrm{cm}^{-1}\right)$ 27,889. FTIR (pure) $v\left(\mathrm{~cm}^{-1}\right): 3514,3366,1643,1590 .{ }^{1} \mathrm{H}-\mathrm{NMR}(300 \mathrm{MHz}$, DMSO- $\left.d_{6}\right) \delta: 7.82(\mathrm{dd}, J=2.0 \mathrm{~Hz}, 8.4 \mathrm{~Hz}, 1 \mathrm{H}, \mathrm{H} 9), 7.77(\mathrm{~d}, J=15.5 \mathrm{~Hz}, 1 \mathrm{H}, \mathrm{H} 2), 7.62$ $(\mathrm{d}, J=15.3 \mathrm{~Hz}, 1 \mathrm{H}, \mathrm{H} 3), 7.60(\mathrm{~d}, J=1.9 \mathrm{~Hz}, 1 \mathrm{H}, \mathrm{H} 13), 7.18\left(\mathrm{~s}, 2 \mathrm{H}, \mathrm{H} 5-5^{\prime}\right), 6.91(\mathrm{~d}, J=8.3 \mathrm{~Hz}$, $1 \mathrm{H}, \mathrm{H} 10), 3.86$ (s, 3H, H15), 3.84 (s, 6H, H14-14'). ${ }^{13} \mathrm{C}-\mathrm{NMR}$ (75 MHz, DMSO-d $d_{6}$ ) $\delta: 186.95$ (C1), 151.81 (C11), 148.09 (C6-6'), 147.85 (C12), 143.97 (C3), 138.44 (C7), 129.77 (C8), 125.29 (C4), 123.70 (C9), 119.02 (C2), 114.87 (C10), 111.53 (C13), 106.78 (C5-5'), 56.22 (C14-14'), 55.71 (C15). HRMS: $m / z[\mathrm{M}+\mathrm{H}]^{+}$calculated for $\mathrm{C}_{18} \mathrm{H}_{19} \mathrm{O}_{6}$ : 331.1181 found: 331.1181.

4, $4^{\prime}$-dihydroxy-3, ${ }^{\prime}, 5,5^{\prime}$-tetramethoxy-chalcone (a12): purification (cyclohexane/ethyl acetate: 50/50) led to a yellow powder (52\%). m.p. $187-188{ }^{\circ} \mathrm{C}, \mathrm{UV}: \lambda_{\max }(\mathrm{EtOH}, \mathrm{nm})$ 377, $\varepsilon\left(\mathrm{L} \cdot \mathrm{mol}^{-1} \cdot \mathrm{cm}^{-1}\right) 30,047$. FTIR (pure) $\vee\left(\mathrm{cm}^{-1}\right)$ : 3304, 1749, 1633, 1582. ${ }^{1} \mathrm{H}-\mathrm{NMR}$ $\left(300 \mathrm{MHz}, \mathrm{DMSO}-d_{6}\right) \delta: 7.77(\mathrm{~d}, J=15.5 \mathrm{~Hz}, 1 \mathrm{H}, \mathrm{H} 2), 7.65(\mathrm{~d}, J=15.3 \mathrm{~Hz}, 1 \mathrm{H}, \mathrm{H} 3), 7.44(\mathrm{~s}, 2 \mathrm{H}$, H9-9'), 7.20 (s, 2H, H5-5'), 3.88 (s, 6H, H13-13'), 3.85 (s, 6H, H12-12'). ${ }^{13} \mathrm{C}-\mathrm{NMR}$ (75 MHz, DMSO- $\left.d_{6}\right) \delta: 187.1(\mathrm{C} 1), 148.09$ (C6-6'), $147.34\left(\mathrm{C} 10-10^{\prime}\right), 144.26$ (C3), 141.07 (C11), 138.57 (C7), 128.44 (C8), 125.27 (C4), 119.12 (C2), 107.05 (C5-5'), 106.83 (C9-9'), 56.39 (C13-13'), 56.28 (C12-12'). HRMS: $m / z[\mathrm{M}+\mathrm{H}]^{+}$calculated for $\mathrm{C}_{19} \mathrm{H}_{21} \mathrm{O}_{7}: 361.1287$ found: 361.1290.

\subsection{General Procedure for Dihydrochalcones Syntheses}

All DHCs were obtained by catalytic hydrogenation of the corresponding $\mathrm{CH}$. The $\mathrm{CH}(400 \mathrm{mg})$ was solubilized in $\mathrm{EtOH}(100 \mathrm{~mL}), 10 \mathrm{wt} . \%$ of palladium on carbon $(\mathrm{Pd} / \mathrm{C})$, was added and the mixture was stirred for a few minutes under nitrogen to remove air before being placed under a continuous flow of $\mathrm{H}_{2}$ with vigorous stirring (to form a vortex) for $45 \mathrm{~min}$. Then, the reaction was replaced under nitrogen to remove residual $\mathrm{H}_{2}$ for a few minutes. The reaction mixture was filtered on a sinter filter with Celite ${ }^{\circledR}$ to remove the $\mathrm{Pd} / \mathrm{C}$. The solvent was evaporated under reduced pressure and the product was dry-loaded onto Celite ${ }^{\circledR}$ to be purified through flash chromatography on silica gel.

For each compound, the numbers for the NMR assignment are related to the labeling in the NMR spectra in the Supplementary Materials.

4,4'-dihydroxy-dihydrochalcone (b1): purification led to a white powder (61\%). m.p. 161$163{ }^{\circ} \mathrm{C}$, UV: $\lambda_{\max }(\mathrm{EtOH}, \mathrm{nm}) 279, \varepsilon\left(\mathrm{L} \cdot \mathrm{mol}^{-1} \cdot \mathrm{cm}^{-1}\right)$ 22,800. FTIR (pure) $v\left(\mathrm{~cm}^{-1}\right): 3417$, 3121, 1652. ${ }^{1} \mathrm{H}-\mathrm{NMR}\left(300 \mathrm{MHz}\right.$, Acetone- $\left.d_{6}\right) \delta: 7.91\left(\mathrm{~d}, J=8.8 \mathrm{~Hz}, 2 \mathrm{H}, \mathrm{H9}-9^{\prime}\right), 7.10(\mathrm{~d}$, $\left.J=8.4 \mathrm{~Hz}, 2 \mathrm{H}, \mathrm{H} 5-5^{\prime}\right), 6.91\left(\mathrm{~d}, J=8.8 \mathrm{~Hz}, 2 \mathrm{H}, \mathrm{H} 10-10^{\prime}\right), 6.74\left(\mathrm{~d}, J=8.4 \mathrm{~Hz}, 2 \mathrm{H}, \mathrm{H} 6-6^{\prime}\right)$, $3.20(\mathrm{t}, J=7.6 \mathrm{~Hz}, 2 \mathrm{H}, \mathrm{H} 2), 2.89(\mathrm{t}, J=7.5 \mathrm{~Hz}, 2 \mathrm{H}, \mathrm{H} 3) .{ }^{13} \mathrm{C}-\mathrm{NMR}\left(75 \mathrm{MHz}\right.$, Acetone- $\left.d_{6}\right) \delta$ : 197.8 (C1), 162.6 (C11), 156.4 (C7), 133.3 (C4), 131.3 (C9-9'), 130.3 (C8), 130.2 (C5-5'), 115.99 $\left(\mathrm{C} 6-6^{\prime}\right), 159.96\left(\mathrm{C} 10-10^{\prime}\right), 40.8(\mathrm{C} 2), 30.2(\mathrm{C} 3)$. HRMS: $m / z[\mathrm{M}+\mathrm{H}]^{+}$calculated for $\mathrm{C}_{15} \mathrm{H}_{15} \mathrm{O}_{3}$ : 243.1021 found: 243.1024 .

3,4,4'-trihydroxy-dihydrochalcone (b2): purification (cyclohexane/ethyl acetate: 55/45) led to a brownish solid (69\%). m.p. $135-136^{\circ} \mathrm{C}, \mathrm{UV}: \lambda_{\max }(\mathrm{EtOH}, \mathrm{nm}) 281, \varepsilon\left(\mathrm{L} \cdot \mathrm{mol}^{-1} \cdot \mathrm{cm}^{-1}\right)$ 21,157. FTIR (pure) $v\left(\mathrm{~cm}^{-1}\right): 3350,1639 .{ }^{1} \mathrm{H}-\mathrm{NMR}\left(300 \mathrm{MHz}\right.$, Acetone- $\left.d_{6}\right) \delta: 7.92(\mathrm{~d}, J=8.7 \mathrm{~Hz}$, 2H, H11-11'), $6.91\left(\mathrm{~d}, J=8.7 \mathrm{~Hz}, 2 \mathrm{H}, \mathrm{H} 12-12^{\prime}\right), 6.76(\mathrm{~d}, J=1.7 \mathrm{~Hz}, 1 \mathrm{H}, \mathrm{H} 5), 6.72(\mathrm{~d}, J=8.0 \mathrm{~Hz}$, $1 \mathrm{H}, \mathrm{H8}), 6.60(\mathrm{dd}, J=1.6 \mathrm{~Hz}, 8.0 \mathrm{~Hz}, 1 \mathrm{H}, \mathrm{H9}), 3.18(\mathrm{t}, J=7.6 \mathrm{~Hz}, 2 \mathrm{H}, \mathrm{H} 2), 2.84(\mathrm{t}, J=7.6 \mathrm{~Hz}, 2 \mathrm{H}$, H3). ${ }^{13} \mathrm{C}-\mathrm{NMR}$ (75 MHz, Acetone- $d_{6}$ ) $\delta: 197.8$ (C1), 162.5 (C13), 145.8 (C6), 144.0 (C7), 134.3 
(C4), 131.3 (C11-11'), 130.3 (C10), 120.4 (C9), 116.4 (C5), 116.0 (C12-12' and C8), 40.7 (C2), 30.4 (C3). HRMS: $m / z$ [M+H] $]^{+}$calculated for $\mathrm{C}_{15} \mathrm{H}_{15} \mathrm{O}_{4}: 259.0970$ found: 259.0965.

$3^{\prime}, 4,4^{\prime}$-trihydroxy-dihydrochalcone (b3): purification (cyclohexane/ethyl acetate: 65/35) led to a white powder $(58 \%)$. m.p. $159-160{ }^{\circ} \mathrm{C}$, UV: $\lambda_{\max }(\mathrm{EtOH}, \mathrm{nm}) 278, \varepsilon\left(\mathrm{L} \cdot \mathrm{mol}^{-1} \cdot \mathrm{cm}^{-1}\right)$ 15,921 . FTIR (pure) $v\left(\mathrm{~cm}^{-1}\right): 3233,3133,1650 .{ }^{1} \mathrm{H}-\mathrm{NMR}\left(300 \mathrm{MHz}\right.$, Acetone- $\left.d_{6}\right) \delta: 7.47(\mathrm{~m}, 2 \mathrm{H}$, $\mathrm{H} 13$ and H9), $7.10(\mathrm{~d}, J=8.4 \mathrm{~Hz}, 2 \mathrm{H}, \mathrm{H} 5-5), 6.89(\mathrm{~d}, J=8.1 \mathrm{~Hz}, 1 \mathrm{H}, \mathrm{H} 10), 6.74(\mathrm{~d}, J=8.4 \mathrm{~Hz}, 2 \mathrm{H}$, H6- $\left.6^{\prime}\right), 3.17(\mathrm{t}, J=7.6 \mathrm{~Hz}, 2 \mathrm{H}, \mathrm{H} 2), 2.88(\mathrm{t}, J=7.6 \mathrm{~Hz}, 2 \mathrm{H}, \mathrm{H} 3) .{ }^{13} \mathrm{C}-\mathrm{NMR}\left(75 \mathrm{MHz}\right.$, Acetone- $\left.d_{6}\right)$ ס: 197.9 (C1), 156.4 (C7), 150.9 (C11), 145.8 (C12), 133.3 (C4), 130.8 (C8), 130.2 (C5-5'), 122.5 (C9), $116.0\left(\mathrm{C}^{-}-6^{\prime}\right), 115.6$ (C10 and C13), 40.7 (C2), 30.3 (C3). HRMS: $m / z[\mathrm{M}+\mathrm{H}]^{+}$calculated for $\mathrm{C}_{15} \mathrm{H}_{15} \mathrm{O}_{4}$ : 259.0970 found: 259.0972 .

$3,3^{\prime}, 4,4^{\prime}$-tetrahydroxy-dihydrochalcone (b4): purification (cyclohexane/ethyl acetate: $60 / 40$ ) led to a white powder $(69 \%)$. m.p. $186-188^{\circ} \mathrm{C}, \mathrm{UV}: \lambda_{\max }(\mathrm{EtOH}, \mathrm{nm}) 280, \varepsilon\left(\mathrm{L} \cdot \mathrm{mol}^{-1} \cdot \mathrm{cm}^{-1}\right)$ 15,752. FTIR (pure) $v\left(\mathrm{~cm}^{-1}\right): 3422,3280,3220,1638 .{ }^{1} \mathrm{H}-\mathrm{NMR}\left(300 \mathrm{MHz}\right.$, Acetone- $\left.d_{6}\right) \delta$ : $7.47(\mathrm{~m}, 2 \mathrm{H}, \mathrm{H} 15$ and H11), $6.89(\mathrm{~d}, J=8.1 \mathrm{~Hz}, 1 \mathrm{H}, \mathrm{H} 12), 6.75(\mathrm{~d}, J=1.7 \mathrm{~Hz}, 1 \mathrm{H}, \mathrm{H} 5), 6.72(\mathrm{~d}$, $J=8.0 \mathrm{~Hz}, 1 \mathrm{H}, \mathrm{H} 8), 6.59(\mathrm{dd}, J=1.7 \mathrm{~Hz}, 8.0 \mathrm{~Hz}, 1 \mathrm{H}, \mathrm{H} 9), 3.15(\mathrm{t}, J=7.6 \mathrm{~Hz}, 2 \mathrm{H}, \mathrm{H} 2), 2.83(\mathrm{t}$, $J=7.6 \mathrm{~Hz}, 2 \mathrm{H}, \mathrm{H3}) .{ }^{13} \mathrm{C}-\mathrm{NMR}\left(75 \mathrm{MHz}\right.$, Acetone- $\left.d_{6}\right) \delta: 197.9$ (C1), 150.9 (C13), 145.8 (C14), 145.7 (C16), 144.0 (C7), 134.3 (C4), 130.8 (C10), 122.5 (C11), 120.4 (C9), 116.4 (C5), 116.0 (C8), 115.64 (C12 or C15), 115.62 (C12 or C15), 40.7 (C2), 30.5 (C3). HRMS: $m / z[\mathrm{M}+\mathrm{H}]^{+}$ calculated for $\mathrm{C}_{15} \mathrm{H}_{15} \mathrm{O}_{5}$ : 275.0919 found: 275.0918 .

4,4'-dihydroxy-3-methoxy-dihydrochalcone (b5): purification (cyclohexane/ethyl acetate: 55/45) led to a white powder (64\%). m.p. $142-143{ }^{\circ} \mathrm{C}$, UV: $\lambda_{\max }(\mathrm{EtOH}, \mathrm{nm}) 280, \varepsilon$ $\left(\mathrm{L} \cdot \mathrm{mol}^{-1} \cdot \mathrm{cm}^{-1}\right) 17,997$. FTIR (pure) $v\left(\mathrm{~cm}^{-1}\right): 3317,1656 .{ }^{1} \mathrm{H}-\mathrm{NMR}\left(300 \mathrm{MHz}\right.$, Acetone- $\left.d_{6}\right)$

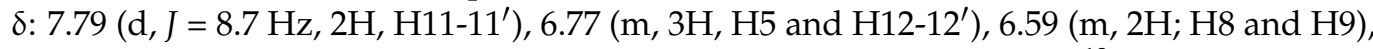
$3.69(\mathrm{~s}, 3 \mathrm{H}, \mathrm{H} 14), 3.10(\mathrm{t}, J=7.7 \mathrm{~Hz}, 2 \mathrm{H}, \mathrm{H} 2), 2.77(\mathrm{t}, J=7.6 \mathrm{~Hz}, 2 \mathrm{H}, \mathrm{H} 3) .{ }^{13} \mathrm{C}-\mathrm{NMR}(75 \mathrm{MHz}$, Acetone- $\left.d_{6}\right)$ 8: 197.9 (C1), 162.6 (C13), 148.2 (C6), 145.6 (C7), 133.9 (C4), 131.3 (C11-11'), 130.3 (C10), 121.6 (C9), 116.0 (C12-12'), 115.6 (C8), 122.9 (C5), 56.2 (C14), 40.8 (C2), 30.7 (C3). HRMS: $m / z[\mathrm{M}+\mathrm{H}]^{+}$calculated for $\mathrm{C}_{16} \mathrm{H}_{17} \mathrm{O}_{4}: 273.1127$ found: 273.1126 .

4,4'-dihydroxy-3'-methoxy-dihydrochalcone (b6): purification (cyclohexane/ethyl acetate: 70/30) led to a white powder (56\%). m.p. $150-151{ }^{\circ} \mathrm{C}, \mathrm{UV}: \lambda_{\max }(\mathrm{EtOH}, \mathrm{nm}) 277, \varepsilon$ $\left(\mathrm{L} \cdot \mathrm{mol}^{-1} \cdot \mathrm{cm}^{-1}\right) 14,793$. FTIR (pure) $v\left(\mathrm{~cm}^{-1}\right): 3320,1658 .{ }^{1} \mathrm{H}-\mathrm{NMR}\left(300 \mathrm{MHz}\right.$, Acetone- $\left.d_{6}\right)$ $\delta: 7.60(\mathrm{dd}, J=1.9 \mathrm{~Hz}, 8.3 \mathrm{~Hz}, 1 \mathrm{H}, \mathrm{H} 9), 7.55(\mathrm{~d}, J=1.7 \mathrm{~Hz}, 1 \mathrm{H}, \mathrm{H} 13), 7.11(\mathrm{~d}, J=8.4 \mathrm{~Hz}, 2 \mathrm{H}$, H5-5'), 6.90 (d, J = 8.2 Hz, 1H, H10), $6.74\left(\mathrm{~d}, J=8.5 \mathrm{~Hz}, 2 \mathrm{H}, \mathrm{H6}-6^{\prime}\right), 3.90$ (s, 3H, H14), $3.22(\mathrm{t}$, $J=7.6 \mathrm{~Hz}, 2 \mathrm{H}, \mathrm{H} 2), 2.90(\mathrm{t}, J=7.6 \mathrm{~Hz}, 2 \mathrm{H}, \mathrm{H} 3) .{ }^{13} \mathrm{C}-\mathrm{NMR}\left(75 \mathrm{MHz}\right.$, Acetone- $\left.d_{6}\right) \delta: 197.8$ (C1), 156.4 (C7), 152.2 (C11), 148.3 (C12), 133.3 (C4), 130.5 (C8), 130.2 (C5-5'), 123.8 (C9), $116.0\left(\mathrm{C} 6-6^{\prime}\right), 115.4$ (C10), 111.5 (C13), 56.3 (C14), 40.7 (C2), 30.3 (C3). HRMS: $m / z$ [M+H] ${ }^{+}$ calculated for $\mathrm{C}_{16} \mathrm{H}_{17} \mathrm{O}_{4}$ : 273.1127 found: 273.1124 .

3,4,4'-trihydroxy-3-methoxy-dihydrochalcone (b7): purification (cyclohexane/ethyl acetate: $70 / 30)$ led to a white powder $(75 \%)$. m.p. $128-130{ }^{\circ} \mathrm{C}, \mathrm{UV}: \lambda_{\max }(\mathrm{EtOH}, \mathrm{nm}) 280$, $\varepsilon\left(\mathrm{L} \cdot \mathrm{mol}^{-1} \cdot \mathrm{cm}^{-1}\right)$ 15,611. FTIR (pure) $v\left(\mathrm{~cm}^{-1}\right): 3484,3356,1651 .{ }^{1} \mathrm{H}-\mathrm{NMR}(300 \mathrm{MHz}$, Acetone- $\left.d_{6}\right) \delta: 7.60(\mathrm{dd}, J=1.9 \mathrm{~Hz}, 8.3 \mathrm{~Hz}, 1 \mathrm{H}, \mathrm{H} 11), 7.56(\mathrm{~d}, J=1.7 \mathrm{~Hz}, 1 \mathrm{H}, \mathrm{H} 15), 6.90$ $(\mathrm{d}, J=8.2 \mathrm{~Hz}, 1 \mathrm{H}, \mathrm{H} 12), 6.76(\mathrm{~d}, J=1.7 \mathrm{~Hz}, 1 \mathrm{H}, \mathrm{H} 5), 6.72(\mathrm{~d}, J=8.0 \mathrm{~Hz}, 1 \mathrm{H}, \mathrm{H} 8), 6.60(\mathrm{dd}$, $J=1.8 \mathrm{~Hz}, 8.0 \mathrm{~Hz}, 1 \mathrm{H}, \mathrm{H9}), 3.90(\mathrm{~s}, 3 \mathrm{H}, \mathrm{H} 16), 3.20(\mathrm{t}, J=7.6 \mathrm{~Hz}, 2 \mathrm{H}, \mathrm{H} 2), 2.84(\mathrm{t}, J=7.6 \mathrm{~Hz}$, 2H, H3). ${ }^{13} \mathrm{C}-\mathrm{NMR}\left(75 \mathrm{MHz}\right.$, Acetone- $d_{6}$ ) $\delta: 197.9$ (C11), 152.2 (C13), 148.3 (C14), 145.8 (C6), 144.0 (C7), 134.3 (C4), 130.6 (C10), 123.8 (C11), 120.5 (C9), 116.4 (C5), 116.0 (C8), 115.4 (C12), 111.6 (C15), 56.3 (C16), $40.7(\mathrm{C} 2), 30.5$ (C3). HRMS: $m / z[\mathrm{M}+\mathrm{H}]^{+}$calculated for $\mathrm{C}_{16} \mathrm{H}_{17} \mathrm{O}_{5}$ : 289.1076 found: 289.1075 .

$3^{\prime}, 4, \mathbf{4}^{\prime}$-trihydroxy-3-methoxy-dihydrochalcone (b8): purification (cyclohexane/ethyl acetate: $70 / 30)$ led to a white powder (46\%). m.p. $139-141{ }^{\circ} \mathrm{C}, \mathrm{UV}: \lambda_{\max }(\mathrm{EtOH}, \mathrm{nm}) 276$, $\varepsilon\left(\mathrm{L} \cdot \mathrm{mol}^{-1} \cdot \mathrm{cm}^{-1}\right)$ 13,187. FTIR (pure) $v\left(\mathrm{~cm}^{-1}\right): 3507,3326,1658 .{ }^{1} \mathrm{H}-\mathrm{NMR}(300 \mathrm{MHz}$, Acetone- $\left.d_{6}\right) \delta: 7.53(\mathrm{~d}, J=1.9 \mathrm{~Hz}, 1 \mathrm{H}, \mathrm{H} 15), 7.47(\mathrm{dd}, J=2.0 \mathrm{~Hz}, 8.3 \mathrm{~Hz}, 1 \mathrm{H}, \mathrm{H} 11), 6.90$ $(\mathrm{d}, J=8.4 \mathrm{~Hz}, 1 \mathrm{H}, \mathrm{H} 12), 6.88(\mathrm{~d}, J=1.2 \mathrm{~Hz}, 1 \mathrm{H}, \mathrm{H} 5), 6.74(\mathrm{~d}, J=8.0 \mathrm{~Hz}, 1 \mathrm{H}, \mathrm{H} 8), 6.70(\mathrm{dd}$, $J=1.4 \mathrm{~Hz}, 8.0 \mathrm{~Hz}, 1 \mathrm{H}, \mathrm{H} 9), 3.80(\mathrm{~s}, 3 \mathrm{H}, \mathrm{H} 16), 3.20(\mathrm{t}, J=7.6 \mathrm{~Hz}, 2 \mathrm{H}, \mathrm{H} 2), 2.89(\mathrm{t}, J=7.6 \mathrm{~Hz}$, 
2H, H3). ${ }^{13} \mathrm{C}-\mathrm{NMR}$ (75 MHz, Acetone- $d_{6}$ ) $\delta: 198.2$ (C1), 150.8 (C12), 148.1 (C6), 145.7 (C14), 145.5 (C7), 133.9 (C4), 130.7 (C10), 122.5 (C11), 121.5 (C9), 115.6 (C12 and C8), 115.5 (C15), 112.8 (C5), 56.1 (C16), 40.7 (C2), 30.6 (C3). HRMS: $m / z[\mathrm{M}+\mathrm{H}]^{+}$calculated for $\mathrm{C}_{16} \mathrm{H}_{17} \mathrm{O}_{5}$ : 289.1076 found: 289.1073 .

4, $4^{\prime}$-dihydroxy-3' ' $^{\prime}$-dimethoxy-dihydrochalcone (b9): purification (cyclohexane/et hyl acetate: $60 / 40$ ) led to a white powder $(47 \%)$. m.p. $149-150{ }^{\circ} \mathrm{C}, \mathrm{UV}: \lambda_{\max }(\mathrm{EtOH}, \mathrm{nm}) 300, \varepsilon$ $\left(\mathrm{L} \cdot \mathrm{mol}^{-1} \cdot \mathrm{cm}^{-1}\right) 19,279$. FTIR (pure) $v\left(\mathrm{~cm}^{-1}\right): 3372,1650 .{ }^{1} \mathrm{H}-\mathrm{NMR}\left(300 \mathrm{MHz}\right.$, Acetone- $\left.d_{6}\right)$

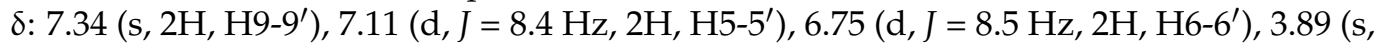
$\left.6 \mathrm{H}, \mathrm{H} 12-12^{\prime}\right), 3.25$ (t, $\left.J=7.6 \mathrm{~Hz}, 2 \mathrm{H}, \mathrm{H} 2\right), 2.90(\mathrm{t}, J=7.6 \mathrm{~Hz}, 2 \mathrm{H}, \mathrm{H} 3) .{ }^{13} \mathrm{C}-\mathrm{CMR}(75 \mathrm{MHz}$, Acetone- $\left.d_{6}\right)$ 8: $197.1(\mathrm{C} 1), 155.6(\mathrm{C} 7), 147.5\left(\mathrm{C} 10-10^{\prime}\right), 140.9$ (C11), 132.4 (C4), 129.4 (C5-5'), 128.1 (C10), 115.1 (C6-6'), 105.9 (C9-9'), 55.8 (C12-12'), 39.8 (C2), 29.7 (C3). HRMS: $m / z$ $[\mathrm{M}+\mathrm{H}]^{+}$calculated for $\mathrm{C}_{17} \mathrm{H}_{19} \mathrm{O}_{5}: 303.1232$ found: 303.1232 .

4, $\mathbf{4}^{\prime}$-dihydroxy-3, $\mathbf{3}^{\prime}$-dimethoxydihydrochalcone (b10): purification (cyclohexane/ethyl acetate: 80/20) led to a white powder (59\%). m.p. $108-109{ }^{\circ} \mathrm{C}, \mathrm{UV}: \lambda_{\max }(\mathrm{EtOH}, \mathrm{nm}) 280, \varepsilon$ $\left(\mathrm{L} \cdot \mathrm{mol}^{-1} \cdot \mathrm{cm}^{-1}\right) 12,962$. FTIR (pure) $\vee\left(\mathrm{cm}^{-1}\right): 3457,3269,1646 .{ }^{1} \mathrm{H}-\mathrm{NMR}(300 \mathrm{MHz}$, Acetone$\left.d_{6}\right) \delta: 7.60(\mathrm{dd}, J=2.0 \mathrm{~Hz}, 8.2 \mathrm{~Hz}, 1 \mathrm{H}, \mathrm{H} 11), 7.55$ (d, J = 2.0 Hz, 1H, H15), 6.90 (s, 1H, H5), 6.89 (d, $J=8.2 \mathrm{~Hz}, 1 \mathrm{H}, \mathrm{H} 12), 6.72$ (m, 2H, H8 and H9), 3.90 (s, 3H, H17), 3.82 (s, 3H, H16), 3.24 $(\mathrm{t}, J=7.6 \mathrm{~Hz}, 2 \mathrm{H}, \mathrm{H} 2), 2.90(\mathrm{t}, J=7.7 \mathrm{~Hz}, 2 \mathrm{H}, \mathrm{H} 3) .{ }^{13} \mathrm{C}-\mathrm{NMR}\left(75 \mathrm{MHz}\right.$, Acetone- $\left.d_{6}\right) \delta: 197.9$ (C1), 152.2 (C13), 148.3 (C14), 148.2 (C6), 145.6 (C7), 133.9 (C4), 130.5 (C10), 123.8 (C11), 121.6 (C9), 115.6 (C8), 115.4 (C12), 112.9 (C5), 111.5 (C15), 56.3 (C17), 56.2 (C16), 40.7 (C2), 30.8 (C3). HRMS: $m / z$ [M+H] $]^{+}$calculated for $\mathrm{C}_{17} \mathrm{H}_{19} \mathrm{O}_{5}: 303.1232$ found: 303.1231 .

4, $\mathbf{4}^{\prime}$-dihydroxy-3,3' ${ }^{\prime}$ 5-trimethoxy-dihydrochalcone (b11): purification (cyclohexane/ ethyl acetate: 60/40) led to a white powder (59\%). m.p. $127-128^{\circ} \mathrm{C}, \mathrm{UV}: \lambda_{\max }(\mathrm{EtOH}, \mathrm{nm}) 276$, $\varepsilon\left(\mathrm{L} \cdot \mathrm{mol}^{-1} \cdot \mathrm{cm}^{-1}\right)$ 13,271. FTIR (pure) $v\left(\mathrm{~cm}^{-1}\right): 3538,3451,1673 .{ }^{1} \mathrm{H}-\mathrm{NMR}(300 \mathrm{MHz}$, Acetone- $\left.d_{6}\right) \delta: 7.60(\mathrm{dd}, J=2.0 \mathrm{~Hz}, 8.3 \mathrm{~Hz}, 1 \mathrm{H}, \mathrm{H} 9), 7.55(\mathrm{~d}, J=2.0 \mathrm{~Hz}, 1 \mathrm{H}, \mathrm{H} 13), 6.90(\mathrm{~d}$, $J=8.6 \mathrm{~Hz}, 1 \mathrm{H}, \mathrm{H} 10), 6.58\left(\mathrm{~s}, 2 \mathrm{H}, \mathrm{H} 5-5^{\prime}\right), 3.90$ (s, 3H, H15), 3.79 (s, 6H, H14-14'), 3.25 (t, $J=7.7 \mathrm{~Hz}, 2 \mathrm{H}, \mathrm{H} 2), 2.90(\mathrm{t}, J=7.7 \mathrm{~Hz}, 2 \mathrm{H}, \mathrm{H} 3) .{ }^{13} \mathrm{C}-\mathrm{NMR}\left(75 \mathrm{MHz}\right.$, Acetone- $\left.d_{6}\right) \delta: 198.0$ (C1), 152.2 (C11), 148.6 (C6-6'), 148.3 (C12), 135.1 (C7), 132.9 (C4), 130.6 (C8), 123.8 (C9), 115.4 (C10), 111.6 (C13), 106.8 (C5-5'), 56.6 (C14-14'), 56.3 (C15), 40.8 (C2), 31.4 (C3). HRMS: $m / z[\mathrm{M}+\mathrm{H}]^{+}$calculated for $\mathrm{C}_{18} \mathrm{H}_{21} \mathrm{O}_{6}: 333.1338$ found: 333.1334 .

4, $4^{\prime}$-dihydroxy-3, $\mathbf{3}^{\prime}, \mathbf{5}, \mathbf{5}^{\prime}$-tetramethoxy-dihydrochalcone (b12): purification (cyclohexane/ ethyl acetate: $60 / 40)$ led to a light brown powder $(38 \%)$. m.p. $168-172{ }^{\circ} \mathrm{C}, \mathrm{UV}: \lambda_{\max }(\mathrm{EtOH}$,

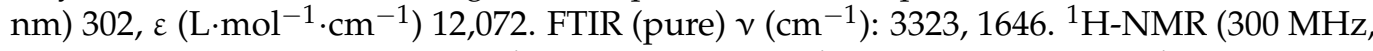
Acetone- $\left.d_{6}\right) \delta: 7.32\left(\mathrm{~s}, 2 \mathrm{H}, \mathrm{H} 9-9^{\prime}\right), 6.58\left(\mathrm{~s}, 2 \mathrm{H}, \mathrm{H} 5-5^{\prime}\right), 3.86\left(\mathrm{~s}, 6 \mathrm{H}, \mathrm{H} 13-13^{\prime}\right), 3.79(\mathrm{~s}, 6 \mathrm{H}$, H12-12'), 3.27 (t, $J=7.6 \mathrm{~Hz}, 2 \mathrm{H}, \mathrm{H} 2), 2.90(\mathrm{t}, J=7.6 \mathrm{~Hz}, 2 \mathrm{H}, \mathrm{H} 3) .{ }^{13} \mathrm{C}-\mathrm{NMR}(75 \mathrm{MHz}$, Acetone- $\left.d_{6}\right)$ 8: $198.1(\mathrm{C} 1), 148.6$ (C6-6'), $148.4\left(\mathrm{C} 10-10^{\prime}\right), 141.8$ (C11), 135.1 (C7), 132.9 (C4), 129.0 (C8), $106.9\left({\left.\mathrm{C} 5-5^{\prime}\right)}^{\prime}, 106.8\left(\mathrm{C}_{-1} 9^{\prime}\right), 59.7\left(\mathrm{C} 13-13^{\prime}\right), 59.6\right.$ (C12-12'), 40.8 (C2), 31.4 (C3). HRMS: $m / z[\mathrm{M}+\mathrm{H}]^{+}$calculated for $\mathrm{C}_{19} \mathrm{H}_{23} \mathrm{O}_{7}: 363.1444$ found: 363.1446 .

\subsection{DPPH Assays}

The radical scavenging activity of all compounds was tested through 2,2-diphenyl-1picryhydrazyl (DPPH) assay which determines the $\mathrm{EC}_{50}$ values as previously described [42]. The test consists of adding $190 \mu \mathrm{L}$ of DPPH solution $(200 \mu \mathrm{M})$ in ethanol to $10 \mu \mathrm{L}$ of potential antiradical molecule solution at different concentrations (from 800 to $12.5 \mu \mathrm{M}$ ). The reaction was carried out in 96-well microplates and the disappearance of DPPH radicals was monitored at $520 \mathrm{~nm}$ every $5 \mathrm{~min}$ during $7.5 \mathrm{~h}$ in a multiplate Biotek (Winooski, VT, USA), Epoch 2 spectrophotometer. The $\mathrm{EC}_{50}$ values, corresponding to the concentration needed to reduce half of the initial population of DPPH radicals, were provided by the crossing point of the curves of \%DPPH and \%reduced DPPH. Curves dots were obtained using an average of the last six measurements for each concentration in Regressi ${ }^{\circledR}$ software version 3.99 . 


\subsection{Anti-Tyrosinase Assays}

Tyrosinase inhibition activity was evaluated using the method used by Peyrot et al. [43]. In a 96-well microplate, $60 \mu \mathrm{L}$ of ammonium formate buffer $(50 \mathrm{mM}, \mathrm{pH}$ 6.4) were mixed with $10 \mu \mathrm{L}$ of inhibitor solution at different concentrations: 10,000, 5000, 1000, 500, 100, 50, $10,5,1$ and $0.5 \mu \mathrm{M}$ in DMSO. Then, $20 \mu \mathrm{L}$ of tyrosine at $4.42 \mathrm{mM}$ in ammonium formate buffer was added. After $10 \mathrm{~s}$ of shaking, $10 \mu \mathrm{L}$ of mushroom tyrosinase $\left(5000 \mathrm{U} \cdot \mathrm{mL}^{-1}\right.$ in ammonium formate buffer) were added and the mixture was incubated for $10 \mathrm{~min}$ at $37^{\circ} \mathrm{C}$. The amount of dopachrome produced during incubation was determined by absorbance readings at $420 \mathrm{~nm}$ every $15 \mathrm{~s}$ in a microplate Biotek (Winooski, VT, USA), Epoch 2 spectrophotometer. $\mathrm{IC}_{50}$ values, corresponding to the concentration required to inhibit $50 \%$ of tyrosinase activity, were obtained using GraphPad Prism ${ }^{\circledR}$ software version 6.01. Kojic acid was used as a reference and all measurements were performed at least in duplicate.

\subsection{UV Analysis and Photostability}

UV-Vis spectra were recorded at $10 \mu \mathrm{M}$ in ethanol on a Cary $60 \mathrm{UV}$-Vis spectrophotometer by Agilent (Wilmington, DE, USA) in a $1 \mathrm{~cm}$ quartz cuvette. The absorbance loss was evaluated by comparison of absorbances at the $\lambda_{\max }$ before and after $1 \mathrm{~h}$ of irradiation into a Rayonet ${ }^{\circledR}$ RPR-200 $\left(\lambda=300 \mathrm{~nm}, \mathrm{P}=8.32 \mathrm{~W} \cdot \mathrm{m}^{-2}\right.$, stirring, $\mathrm{T}=35^{\circ} \mathrm{C}$ ) (SNE Ultraviolet Co. Branford, CT, USA) using 14 RPR-3000A lamps.

\section{Results and Discussion}

\subsection{Synthesis of Chalcones and Dihydrochalcones}

$\mathrm{CHs}$ substituted by phenolic or aromatic methoxy moieties are common in nature and exhibit numerous biological activities [44]. Therefore, we first oriented our choice towards the synthesis of $\mathrm{CHs}$ substituted by $\mathrm{OH}$ in ortho position on the $\mathrm{A}$ ring as is the case for phloretin. However, in accordance with previous studies and our tests, under acid conditions, the formation of the cyclized forms is largely favored. Indeed, in the absence of protection, ortho-OH readily reacted with the double bond of the $\alpha, \beta$-unsaturated ketone system of the $\mathrm{CH}$ resulting from the condensation between the aldehyde and the ketone [45]. Given these preliminary results and our desire to offer a green synthetic pathway, ketones with $\mathrm{OH}$ in ortho position were ruled out. The same observation was done with aldehydes substituted with $\mathrm{OH}$ in ortho, and so they were also ruled out [46].

We then explored the synthesis of $\mathrm{CHs}$ substituted by $\mathrm{OH}$ in para-position on A-ring and B-ring. Indeed, such a substituent in para has already demonstrated diverse biological activities for other molecules $[47,48]$. Furthermore, our preliminary results seemed to show that para-OH promoted the synthesis by increasing electron delocalization and thus making the aldehyde more electrophilic [49]. On the contrary, it was observed that a single $\mathrm{OH}$ in meta position on aldehydes or ketones adversely affects $\mathrm{CHs}$ synthesis. Indeed, these $\mathrm{OH}$ does not allow the delocalization of electrons to the ketone and aldehyde moieties, thus limiting their reactivity. Nevertheless, an additional $\mathrm{OH}$ in the para position allowed the reaction.

Considering the aforementioned observations, in order to offer biobased products and the greenest possible synthetic pathway, we oriented our choice towards aldehydes and ketones substituted by $\mathrm{OH}$ in para-position and simple substituents (-OH or $-\mathrm{OCH}_{3}$ ) in meta-position (Scheme 2, series 0). This way, the ketones chosen were piceol, 3,4-dihydroxyacetopehnone (i.e., acetopyrocatechol), acetovanillone, and acetosyringone, and aldehydes chosen were 4-hydroxybenzaldehyde, 3,4-dihydroxybenzaldehyde (i.e., protocatechualdehyde), vanillin and syringaldehyde (Scheme 2, series 0 ).

Various combinations of these ketones and aldehydes were performed through a green synthesis using ethanol as solvent and a catalytic amount of $\mathrm{HCl}$ to initiate the Claisen-Schmidt reaction. They led to $12 \mathrm{CHs}$ (a1 to a12) in yields between $47 \%$ and $80 \%$ after purification (Scheme 2, series a). 
Once again, the syringol groups on $\mathrm{CHs}$ limited their hydrogenation and led to poor yields of $47 \%$ and $38 \%$ for $\mathbf{b} 9$ and $\mathbf{b} \mathbf{1 2}$, respectively.

Thanks to this simple two-step synthesis, we constituted a library of 24 molecules presenting various substitutions allowing different activities for the cosmetic sector. To the best of our knowledge, seven of them have never been described. All the chemical structures were confirmed by ${ }^{1} \mathrm{H}-\mathrm{NMR},{ }^{13} \mathrm{C}-\mathrm{NRM}$ (Spectra in Supplementary Materials) and HRMS analyses.

\subsection{Antiradical Activities}

All synthesized compounds, CHs and DHCs, were evaluated as potential antioxidants to be used in cosmetics through DPPH assay. Indeed, antioxidants are present in most of the formulations, and they protect the skin against damages from reactive oxygen species (ROS) but also stabilize formulations [51]. While ROS are naturally produced by biochemical reactions in human skin surface or from environmental stimulation, they can be harmful in high concentrations by inducing irreversible cellular damages. They result from molecular oxygen reduction and can lead to oxidative stress, premature skin aging or carcinogenesis [52]. Currently, two petro-sourced compounds are commonly used as antiradical agents, BHA (butylated hydroxyanisol) and BHT (butylated hydroxytoluene) but they are more and more criticized and suspected to be endocrine disruptor and carcinogenic $[53,54]$. Therefore, they need to be quickly replaced by natural, biobased and safe alternatives.

To evaluate the $\mathrm{CH}$ and $\mathrm{DHC}$ s potential as radical scavengers, their $\mathrm{EC}_{50}$ values were determined and compared with that of $\mathrm{BHA}, \mathrm{BHT}$ and phloretin. The lower the $\mathrm{EC}_{50}$, the better the antiradical activity was. Results are summarized in Figure 2.

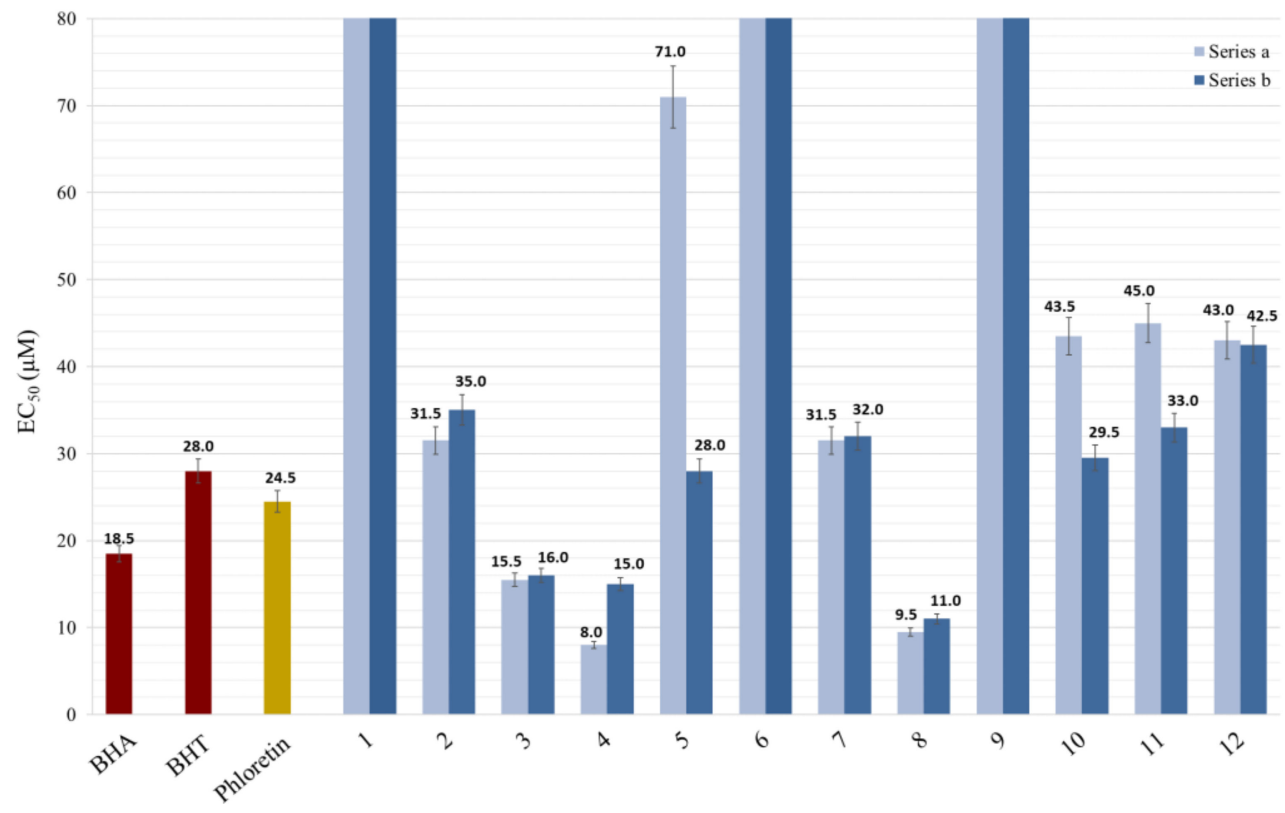

Figure 2. $\mathrm{EC}_{50}$ values (in $\mu \mathrm{M}$ ) of synthetized molecules for DPPH inhibition in ethanol.

As expected, phloretin exhibited a great antioxidant activity $\left(\mathrm{EC}_{50}=24.5 \mu \mathrm{M}\right)$, lower than BHA $\left(\mathrm{EC}_{50}=18.5 \mu \mathrm{M}\right)$, but competitive regarding $\mathrm{BHT}\left(\mathrm{EC}_{50}=28 \mu \mathrm{M}\right)$.

From a global point of view, not all compounds showed antioxidant activity: a1, a6 and their corresponding DHCs $(\mathbf{b} \mathbf{1}, \mathbf{b} 6)$, as well as $\mathbf{a 9}$ and $\mathbf{b 9}$, exhibited $\mathrm{EC}_{50}$ higher than $80 \mu \mathrm{M}$, which means an absence of activity due to the presence of only one hydroxy group on the B-ring in para-position. These results were expected. Indeed, compounds with similar structures, such as $p$-coumaric acid and derivatives, do not present antiradical activity $[42,55]$. However, $\mathbf{a} 3$ and $\mathbf{b} 3$, which were also B-ring-substituted by only one hydroxy group, showed very competitive $\mathrm{EC}_{50}$ values regarding that of phloretin (15.5 and 
$16.0 \mu \mathrm{M}$, respectively). This difference was clearly due to the catechol moiety present at the A-ring level, well described for its antioxidant activity. This observation was confirmed by the low $\mathrm{EC}_{50}$ of other compounds with these patterns $(\mathbf{a} 4, \mathbf{b} 4, \mathbf{a} 8$ and $\mathbf{b 8})$ presenting values of $8,15,9.5$ and $11 \mu \mathrm{M}$, respectively. Catechol moiety on B-ring also provided great antiradical activities as observed for compounds $\mathbf{a} 2, \mathbf{b} 2$, a7 and $\mathbf{b} 7$, which showed interesting values ranging between 31 and $35 \mu \mathrm{M}$. These groups were strongly involved in the antiradical activity, whether they were on A- or B-ring, as has been already demonstrated by Kozlowski et al. [56]. These authors also highlighted the guaiacol moiety contribution (i.e., $\mathrm{OH}$ and $\mathrm{OCH}_{3}$ groups in ortho configuration), especially on the B-ring, which confirmed our observations for compounds a5, b5, a10 and b10. B-ring syringol moieties seemed to also influence the antioxidant activity, but to a lesser extent, as evidenced by the $\mathrm{EC}_{50}$ values of the compounds a11, b11, a12 and b12. As was already observed by Mouterde et al., by comparing the wholeness of $\mathrm{EC}_{50}$ values, an additional hydroxy group impacted more strongly the antioxidant activity than a methoxy [57].

Finally, contrary to what we expected, some DHCs (series b) showed better antioxidant activity than their corresponding $\mathrm{CHs}$ (b5, b10 and b11), even though it was previously observed that the $\alpha, \beta$-double bond is important for the activity [56]. This phenomenon was probably due to the presence of a common guaiacol moiety.

Overall, for both series, the most effective molecules were substituted by catechol groups, and their $\mathrm{EC}_{50}$ values were highly competitive with the currently used references and phloretin. These results may be confirmed in vivo.

\subsection{Anti-Tyrosinase Activities}

Tyrosinase is the key enzyme of skin and hair pigments biosynthesis. Indeed, it intervenes in the two first steps of melanin production. First, it hydroxylates tyrosine into L-DOPA (3,4-dihydroxyphenylalanine); then, it oxidizes L-DOPA into L-DOPAquinone. The latter spontaneously rearranges to form L-DOPAchrome. Melanin protects the skin against UV radiation, but overexposure to the sun can lead to abnormal production and thus to hyperpigmentation on exposed areas. Tyrosinase inhibition is one more common way to reduce pigment spots. For this reason, tyrosinase inhibitors are widely used as lightening agents in cosmetics.

Tests have been performed mimicking human tyrosinase activity using a fungal tyrosinase. Through these tests, the $\mathrm{IC}_{50}$ values of all the synthesized molecules were determined and compared to that of Kojic acid, which is used as a reference for in tubo tyrosinase inhibition [58]. The lower the $\mathrm{IC}_{50}$ value, the better the anti-tyrosinase effect is. Results are summarized in Table 1.

First, contrary to many studies [20,59], in our experimental conditions, phloretin did not exhibit tyrosinase inhibition activity against tyrosinase from mushrooms.

Concerning the synthesized molecules, five $\mathrm{CH}$ showed lower $\mathrm{IC}_{50}$ values than Kojic acid $\left(\mathrm{IC}_{50}=0.42 \mathrm{mM}\right)$. In terms of structure-activity relationship, a para-OH substituent on B-ring led to higher tyrosinase inhibition. Indeed, the $\mathrm{CHs} \mathbf{a 1}$, a9 and $\mathbf{a} 6$ presented the greater activity, with $\mathrm{IC}_{50}$ values of $0.1,0.13$ and $0.14 \mathrm{mM}$, respectively. Surprisingly, the compound a3 did not show any activity, although it was also 4-OH-substituted. Moreover, a catechol group on B-ring also provided enzyme inhibition, such as a2 and a4, which exhibited IC 50 of 0.23 and $0.50 \mathrm{mM}$, respectively, competitive with kojic acid value. Finally, a10, a11 and a7, substituted by a guaiacol group on A-ring, displayed a relatively poor inhibition, indicating that this moiety may decrease the bioactivity.

CHs were overall more efficient than DCHs. Indeed, only three DHCs, b5, b8 and b10, revealed inhibition activity. However, their $\mathrm{IC}_{50}$ values were better than those of Kojic acid, and all had the same B-ring (guaiacol moiety).

Considering these observations, the tyrosinase inhibition activity appeared to be mainly related to the B-ring substitution according to the following order for CHs: hydroxy > catechol $>$ guaiacol. Concerning DHCs, only guaiacol groups induced an activity. Syringol moiety 
did not show any tyrosinase inhibition. Although some compounds are very promising for cosmetics uses, these results should be confirmed subsequently by tests on cells.

Table 1. $\mathrm{IC}_{50}$ of synthesized compounds for mushroom tyrosinase inhibition.

\begin{tabular}{|c|c|c|}
\hline Series & Compound & $\mathrm{IC}_{50}(\mathrm{mM})$ \\
\hline \multirow{12}{*}{ Chalcones series } & a1 & $0.10 \pm 0.01$ \\
\hline & a2 & $0.50 \pm 0.03$ \\
\hline & a3 & $-a$ \\
\hline & a4 & $0.23 \pm 0.01$ \\
\hline & a5 & $2.3 \pm 0.1$ \\
\hline & a6 & $0.14 \pm 0.01$ \\
\hline & a7 & $2.3 \pm 0.1$ \\
\hline & a8 & $-a$ \\
\hline & a9 & $0.13 \pm 0.01$ \\
\hline & a10 & $1.80 \pm 0.09$ \\
\hline & a11 & $7.9 \pm 0.4$ \\
\hline & a12 & $-a$ \\
\hline \multirow{12}{*}{ Dihydrochalcones series } & b1 & $-a$ \\
\hline & b2 & $-a$ \\
\hline & b3 & $-a^{a}$ \\
\hline & b4 & $-a$ \\
\hline & b5 & $0.50 \pm 0.03$ \\
\hline & b6 & $-a$ \\
\hline & b7 & $-{ }^{a}$ \\
\hline & b8 & $0.07 \pm 0.01$ \\
\hline & b9 & $-a$ \\
\hline & b10 & $0.27 \pm 0.01$ \\
\hline & b11 & $-a$ \\
\hline & b12 & $-a$ \\
\hline \multirow{2}{*}{ References } & Phloretin & $-a$ \\
\hline & Kojic acid & $0.42 \pm 0.02$ \\
\hline
\end{tabular}

a No activity observed.

\subsection{UV Analysis and Molecules Photostability}

Currently, the sunscreen market is characterized by a strong demand for new biobased UV filters, especially since petrosourced filters such as avobenzone and octinoxate are increasingly criticized due to their environmental and health impact [60-62]. It is well known that conjugated molecules can absorb ultraviolet wavelengths, so we undertook to examine the potential of our molecules for this application.

To this aim, we first evaluated UV properties by recording UV-Vis spectra at $10 \mu \mathrm{M}$ in ethanol. Regarding the maximum absorption wavelengths obtained for $\mathrm{CHs}$, a1-a12 (350-393 nm), spectra were compared with avobenzone (UV-A reference, $\lambda_{\max }=357 \mathrm{~nm}$ ) (Figure $\left.3 \mathrm{~A}, \mathrm{~A}^{\prime}\right)$. Concerning DHCs b1-b12 and phloretin, the maximum absorption wavelengths $\left(278-302 \mathrm{~nm}\right.$ ) were preferentially compared with octinoxate (UV-B reference, $\lambda_{\max }=310 \mathrm{~nm}$ ) (Figure 3B, $\mathrm{B}^{\prime}$ ).

$\mathrm{CHs}$ presented similar spectra with two major absorption bands. The first in the range $200-250 \mathrm{~nm}$, generally noted as band II, was due to the A-ring benzoyl system. The second one, band I, in the range 340-400 nm originated from the B-ring cinnamoyl system [28] and was close to the avobenzone wavelength coverage (dotted black line, Figure $\left.3 \mathrm{~A}, \mathrm{~A}^{\prime}\right)$. Only three compounds were competitive in regard to avobenzone (0.37): a1, a4 and a7 with absorbance values of $0.65,0.47$ and 0.38 , respectively. Concerning other $\mathrm{CHs}$, their absorbances were equivalent, around $0.29-0.30$, for whom the lowest was 0.27 for compound a11, and thus were far from being insignificant. 

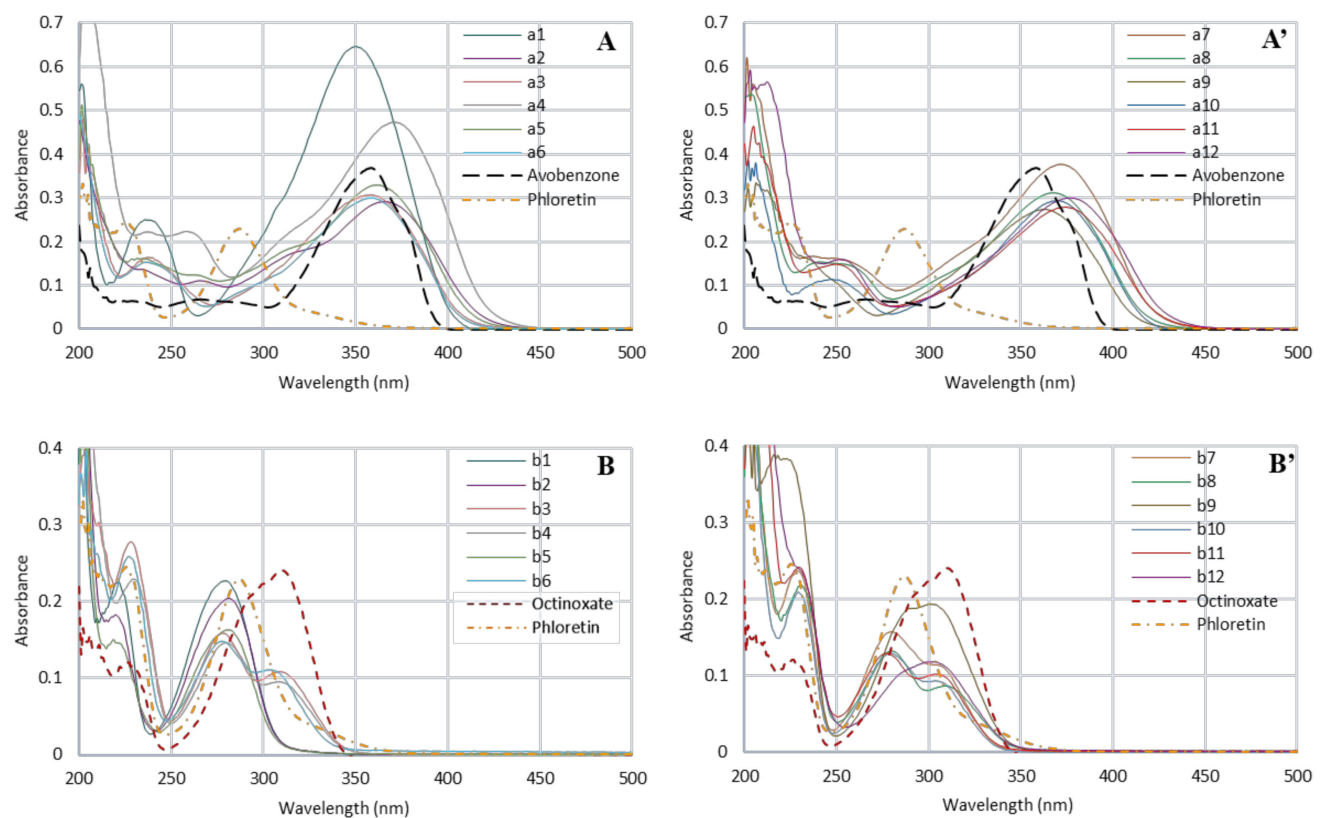

Figure 3. UV-Vis spectrum of chalcones series $\left(\mathbf{A}, \mathbf{A}^{\prime}\right)$, dihydrochalcones series $\left(\mathbf{B}, \mathbf{B}^{\prime}\right)$, phloretin (dotted orange line), avobenzone (dotted black line) and octinoxate (dotted red line) at $10 \mu \mathrm{M}$ in ethanol. For each series, spectra have been split into two graphics and the scale has been adapted for clarity.

Regarding the DHCs (i.e., phloretin and the twelve analogs), the presence of the two bands was also observed, although as expected, the absence of the double bond resulted in a hypsochromic shift of the band I at around $250-350 \mathrm{~nm}$ covering a part of UV-B like with octinoxate (dotted red line, Figure $3 \mathrm{~B}, \mathrm{~B}^{\prime}$ ). DHCs absorbances were lower than those of CHs. However, phloretin showed a maximal absorbance (0.23), competitive with octinoxate (0.24), as did compound $\mathbf{b} \mathbf{1}(0.23)$. Finally, $\mathbf{b} \mathbf{2}$ and $\mathbf{b} \mathbf{9}$ also revealed significant absorbances ( 0.21 and 0.19 , respectively), while the other DHCs were not efficient enough. It may be noted that some compounds presented two absorption maxima for the band I (b3, b4, b6, b7, b8, and b11).

Studying wavelength coverage and absorbance intensity was not enough to evaluate the potential of CHs and DHCs as UV filters. Indeed, it is important to assess their photostability, correlated to their loss of absorbance upon UV irradiations. For this, compounds in ethanol were irradiated $(10 \mu \mathrm{M}, 1 \mathrm{~h}, 300 \mathrm{~nm})$. UV-Vis spectra of the resulting solutions were recorded, and the maximal absorbance were compared to the non-irradiated solutions for each compound. $\mathrm{CHs}$ and phloretin absorbance losses are presented in Figure 4 and those of DHCs in Figure 5 (Spectra in Supplementary Materials).

First, concerning phloretin, its loss of absorbance was $5.9 \%$ against $26.0 \%$ for octinoxate and only $0.6 \%$ for avobenzone. Peyrot et al. mentioned in their study that the acceptable absorbance loss to match the commercial UV filters specifications should be less than $5 \%$ [43]. Phloretin's result was very close to these specifications and revealed itself more photostable than octinoxate. Thus, this natural compound constitutes an alternative to petrosourced UV-B filters.

Regarding the synthesized $\mathrm{CHs}$, promising compounds in terms of absorption intensity, a1, a4 and a7 showed excessively high absorbance losses of $77.6 \%, 75.7 \%$ and $46.2 \%$, respectively. Only a6 displayed a loss of absorbance of $16.1 \%$ that could compete with UV-A filters. 


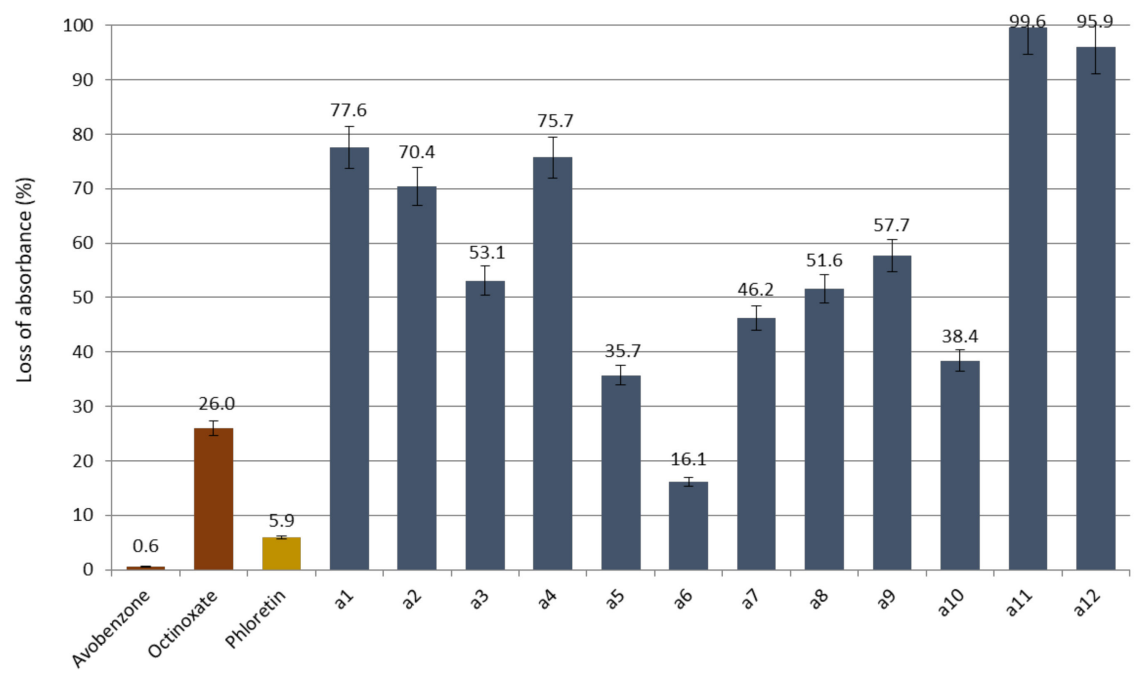

Figure 4. Loss of absorbance (in \%) for chalcone series, phloretin and references avobenzone and octinoxate.

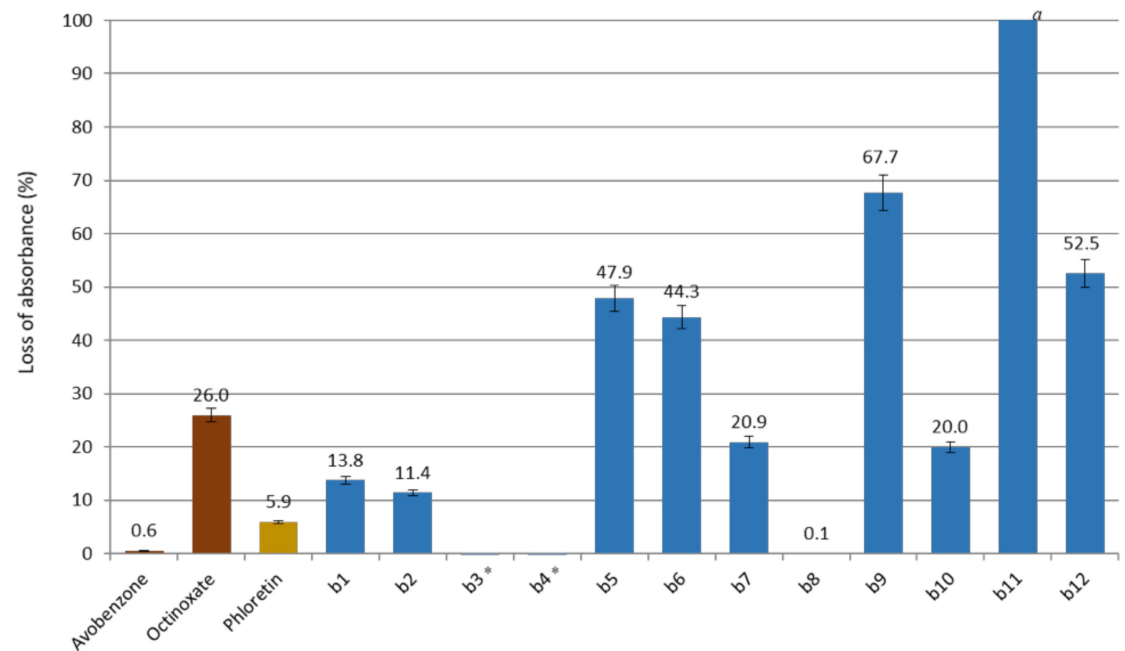

Figure 5. Loss of absorbance (in \%) for dihydrochalcones series, phloretin and references avobenzone and octinoxate. ${ }^{\text {a }}$ Full loss of absorbance. ${ }^{*}$ Negative loss of absorbance (after $1 \mathrm{~h}$ of irradiation the absorbance was higher than at $\mathrm{t} 0$ ).

It is noteworthy to mention that, unexpectedly, DHCs $\mathbf{b} \mathbf{3}$ and $\mathbf{b} \mathbf{4}$ showed a higher absorbance higher after $1 \mathrm{~h}$ of irradiation. Indeed, for these compounds, the exposure to UV induced the disappearance of the second maximum absorption and the increase of the first (see spectra in Supplementary Materials). This was also the case for b8, which presented a very poor loss of absorbance of $0.1 \%$. However, it was not the case for all compounds having two maximum absorption at t0, such as $\mathbf{b} 6$ and b11. There does not seem to be any correlation between the structure of the molecule and the presence of these two maximum absorbances. Nonetheless, compounds b3, b4 and b8 were all A-ring-substituted by a catechol group. A catechol B-ring substitution also provided a relatively low loss of absorbance, as shown by $\mathbf{b} \mathbf{2}$ and $\mathbf{b} 7$ data, which were lower than those of octinoxate.

Unfortunately, b9, which was far from being insignificant in terms of absorbance, showed after irradiation $67.7 \%$ of loss of absorbance. On the contrary, b10, presenting a low loss of absorbance (20\%), had a maximum absorbance at t0 of 0.13 , too low to compete with the current UV filters. 
Finally, $\mathbf{b} \mathbf{1}$ and $\mathbf{b} \mathbf{2}$ were the only two synthesized analogs potentially competing with octinoxate. Indeed, in addition to displaying a maximum absorbance close to that of the reference, they showed a relatively low loss of absorbance $(13.8 \%$ and $11.4 \%$, respectively).

To conclude, $\mathrm{CHs}$ appeared to be non-competitive as UV filters, especially compared to avobenzone which absorbs at the same wavelengths. Concerning DHCs, b1 and b2 appeared as potential alternatives to octinoxate. However, as with any in vitro test, these results remain to be confirmed in vivo.

\section{Conclusions}

In light of the high potential of phloretin in terms of biological activities and considering its low availability and its tedious and non-sustainable chemical synthesis, twenty-four analogs (chalcones and dihydrochalcones) were synthesized in good yields through a green $\mathrm{HCl}$-catalyzed Claisen-Schmidt condensation between biobased ketones and aldehydes in ethanol followed by palladium-catalyzed hydrogenation. Antioxidant, anti-tyrosinase and UV filter properties were investigated for all compounds. Structure-activity relationships revealed the importance of the number and positions of hydroxyl substituents. This study confirmed the potent activity of catechol groups on CHs and DHCs. Finally, even if only two analogs (b1 and b2) showed interesting anti-UV properties, several compounds exhibited promising antioxidant and/or anti-tyrosinase activity, especially $\mathbf{a} 4$ and b8, which appeared as competitive biobased antioxidants and tyrosinase inhibitors and thus sustainable alternatives to petrosourced counterparts. However, they must be more investigated further before a potential application in cosmetics; the results of these investigations will be reported in due course.

Supplementary Materials: The following are available online at https:/ /www.mdpi.com/2076-392 $1 / 10 / 4 / 512 / s 1$, the ${ }^{1} \mathrm{H}$ and ${ }^{13} \mathrm{C}-\mathrm{NMR}$ spectra, tyrosinase inhibitor assay, DPPH assay, UV spectrum and loss of absorbance study are provided for every single molecule synthesized in this work.

Author Contributions: Conceptualization: L.M., C.P., F.A. and J.-H.R.; methodology: L.M.; formal analysis: L.M.; HRMS analysis: F.B.; data curation: L.M. and C.P.; writing original draft preparation: L.M. and C.P.; writing-review and editing: F.A. and C.P.; supervision: C.P., F.A. and J.-H.R.; project administration: F.A. and J.-H.R.; funding acquisition: F.A. All authors have read and agreed to the published version of the manuscript.

Funding: The authors are grateful for funding received from the Interreg North-West Europe program: AgriWasteValue (no. NWE 750) and thank Grand Reims, Conseil Général de la Marne and Région Grand-Est for their financial support.

Institutional Review Board Statement: Not applicable.

Informed Consent Statement: Not applicable.

Data Availability Statement: Data is contained within the article and supplementary material.

Conflicts of Interest: The authors declare no conflict of interest.

\section{Abbreviations}

$\mathrm{CH}(\mathrm{s})$ : chalcone(s); $\mathrm{DHC}(\mathrm{s})$, dihydrochalcone(s).

\section{References}

1. Thompson-Witrick, K.A.; Goodrich, K.M.; Neilson, A.P.; Hurley, E.K.; Peck, G.M.; Stewart, A.C. Characterization of the Polyphenol Composition of 20 Cultivars of Cider, Processing, and Dessert Apples (Malus $\times$ Domestica Borkh.) Grown in Virginia. J. Agric. Food Chem. 2014, 62, 10181-10191. [CrossRef] [PubMed]

2. Picinelli, A.; Dapena, E.; Mangas, J.J. Polyphenolic Pattern in Apple Tree Leaves in Relation to Scab Resistance. A Preliminary Study. J. Agric. Food Chem. 1995, 43, 2273-2278. [CrossRef]

3. Moreira, M.M.; Barroso, M.F.; Boeykens, A.; Withouck, H.; Morais, S.; Delerue-Matos, C. Valorization of Apple Tree Wood Residues by Polyphenols Extraction: Comparison between Conventional and Microwave-Assisted Extraction. Ind. Crops Prod. 2017, 104, 210-220. [CrossRef] 
4. Rana, S.; Rana, A.; Gulati, A.; Bhushan, S. RP-HPLC-DAD Determination of Phenolics in Industrial Apple Pomace. Food Anal. Methods 2014, 7, 1424-1432. [CrossRef]

5. Zielinska, D.; Laparra-Llopis, J.M.; Zielinski, H.; Szawara-Nowak, D.; Giménez-Bastida, J.A. Role of Apple Phytochemicals, Phloretin and Phloridzin, in Modulating Processes Related to Intestinal Inflammation. Nutrients 2019, 11, 1173. [CrossRef]

6. Zhang, Z.; Li, S.; Cao, H.; Shen, P.; Liu, J.; Fu, Y.; Cao, Y.; Zhang, N. The Protective Role of Phloretin against Dextran Sulfate Sodium-Induced Ulcerative Colitis in Mice. Food Funct. 2019, 10, 422-431. [CrossRef]

7. Jung, M.; Triebel, S.; Anke, T.; Richling, E.; Erkel, G. Influence of Apple Polyphenols on Inflammatory Gene Expression. Mol. Nutr. Food Res. 2009, 53, 1263-1280. [CrossRef]

8. Park, S.Y.; Kim, E.J.; Shin, H.-K.; Kwon, D.Y.; Kim, M.S.; Surh, Y.-J.; Park, J.H.Y. Induction of Apoptosis in HT-29 Colon Cancer Cells by Phloretin. J. Med. Food 2007, 10, 581-586. [CrossRef]

9. Yang, K.-C.; Tsai, C.-Y.; Wang, Y.-J.; Wei, P.-L.; Lee, C.-H.; Chen, J.-H.; Wu, C.-H.; Ho, Y.-S. Apple Polyphenol Phloretin Potentiates the Anticancer Actions of Paclitaxel through Induction of Apoptosis in Human Hep G2 Cells. Mol. Carcinog. 2009, 48, $420-431$. [CrossRef] [PubMed]

10. Kern, M.; Pahlke, G.; Balavenkatraman, K.K.; Böhmer, F.D.; Marko, D. Apple Polyphenols Affect Protein Kinase C Activity and the Onset of Apoptosis in Human Colon Carcinoma Cells. J. Agric. Food Chem. 2007, 55, 4999-5006. [CrossRef]

11. Behzad, S.; Sureda, A.; Barreca, D.; Nabavi, S.F.; Rastrelli, L.; Nabavi, S.M. Health Effects of Phloretin: From Chemistry to Medicine. Phytochem. Rev. 2017, 527-533. [CrossRef]

12. Barreca, D.; Bellocco, E.; Laganà, G.; Ginestra, G.; Bisignano, C. Biochemical and Antimicrobial Activity of Phloretin and Its Glycosilated Derivatives Present in Apple and Kumquat. Food Chem. 2014, 160, 292-297. [CrossRef] [PubMed]

13. Darbre, P.D.; Harvey, P.W. Paraben Esters: Review of Recent Studies of Endocrine Toxicity, Absorption, Esterase and Human Exposure, and Discussion of Potential Human Health Risks. J. Appl. Toxicol. 2008, 28, 561-578. [CrossRef]

14. Lee, J.-H.; Regmi, S.C.; Kim, J.-A.; Cho, M.H.; Yun, H.; Lee, C.-S.; Lee, J. Apple Flavonoid Phloretin Inhibits Escherichia Coli O157:H7 Biofilm Formation and Ameliorates Colon Inflammation in Rats. Infect. Immun. 2011, 79, 4819-4827. [CrossRef]

15. Wei, L.N.; Shi, C.Z.; Luo, C.X.; Hu, C.Y.; Meng, Y.H. Phloretin Inhibits Biofilm Formation by Affecting Quorum Sensing under Different Temperature. LWT 2020, 131, 109-668. [CrossRef]

16. Rezk, B.M.; Haenen, G.R.M.M.; van der Vijgh, W.J.F.; Bast, A. The Antioxidant Activity of Phloretin: The Disclosure of a New Antioxidant Pharmacophore in Flavonoids. Biochem. Biophys. Res. Commun. 2002, 295, 9-13. [CrossRef]

17. Ridgway, T.; O’Reilly, J.; West, G.; Tucker, G.; Wiseman, H. Antioxidant Action of Novel Derivatives of the Apple-Derived Flavonoid Phloridzin Compared to Oestrogen: Relevance to Potential Cardioprotective Action. Biochem. Soc. Trans. 1997, 25, 106S. [CrossRef]

18. Cullen, J.K.; Simmons, J.L.; Parsons, P.G.; Boyle, G.M. Topical Treatments for Skin Cancer. Adv. Drug Deliv. Rev. 2020, 153, 54-64. [CrossRef]

19. Sajadimajd, S.; Bahramsoltani, R.; Iranpanah, A.; Kumar Patra, J.; Das, G.; Gouda, S.; Rahimi, R.; Rezaeiamiri, E.; Cao, H.; Giampieri, F.; et al. Advances on Natural Polyphenols as Anticancer Agents for Skin Cancer. Pharmacol. Res. 2020, $151,104584$. [CrossRef] [PubMed]

20. Zhang, L.-Q.; Yang, X.-W.; Zhang, Y.-B.; Zhai, Y.-Y.; Xu, W.; Zhao, B.; Liu, D.-L.; Yu, H.-J. Biotransformation of Phlorizin by Human Intestinal Flora and Inhibition of Biotransformation Products on Tyrosinase Activity. Food Chem. 2012, 132, 936-942. [CrossRef]

21. Zuo, A.-R.; Yu, Y.-Y.; Shu, Q.-L.; Zheng, L.-X.; Wang, X.-M.; Peng, S.-H.; Xie, Y.-F.; Cao, S.-W. Hepatoprotective Effects and Antioxidant, Antityrosinase Activities of Phloretin and Phloretin Isonicotinyl Hydrazone. J. Chin. Med Assoc. 2014, 77, $290-301$. [CrossRef] [PubMed]

22. Oresajo, C.; Stephens, T.; Hino, P.D.; Law, R.M.; Yatskayer, M.; Foltis, P.; Pillai, S.; Pinnell, S.R. Protective Effects of a Topical Antioxidant Mixture Containing Vitamin C, Ferulic Acid, and Phloretin against Ultraviolet-Induced Photodamage in Human Skin. J. Cosmet. Dermatol. 2008, 7, 290-297. [CrossRef] [PubMed]

23. Auner, B.G.; O'Neill, M.A.A.; Valenta, C.; Hadgraft, J. Interaction of Phloretin and 6-Ketocholestanol with DPPC-Liposomes as Phospholipid Model Membranes. Int. J. Pharm. 2005, 294, 149-155. [CrossRef]

24. Siddaiah, V.; Rao, C.V.; Venkateswarlu, S.; Subbaraju, G.V. A Concise Synthesis of Polyhydroxydihydrochalcones and Homoisoflavonoids. Tetrahedron 2006, 62, 841-846. [CrossRef]

25. Sato, S.; Akiya, T.; Nishizawa, H.; Suzuki, T. Total Synthesis of Three Naturally Occurring 6,8-Di-C-Glycosylflavonoids: Phloretin, Naringenin, and Apigenin Bis-C- $\beta$-d-Glucosides. Carbohydr. Res. 2006, 341, 964-970. [CrossRef]

26. Vijaya Bhaskar Reddy, M.; Hung, H.-Y.; Kuo, P.-C.; Huang, G.-J.; Chan, Y.-Y.; Huang, S.-C.; Wu, S.-J.; Morris-Natschke, S.L.; Lee, K.-H.; Wu, T.-S. Synthesis and Biological Evaluation of Chalcone, Dihydrochalcone, and 1,3-Diarylpropane Analogs as Anti-Inflammatory Agents. Bioorg. Med. Chem. Lett. 2017, 27, 1547-1550. [CrossRef] [PubMed]

27. Farooq, S.; Ngaini, Z. Recent Synthetic Methodologies for Chalcone Synthesis (2013-2018). COCAT 2019, 6, 184-192. [CrossRef]

28. Rammohan, A.; Reddy, J.S.; Sravya, G.; Rao, C.N.; Zyryanov, G.V. Chalcone Synthesis, Properties and Medicinal Applications: A Review. Environ. Chem. Lett. 2020, 18, 433-458. [CrossRef]

29. Da Silva, P.T.; da Cunha Xavier, J.; Freitas, T.S.; Oliveira, M.M.; Coutinho, H.D.M.; Leal, A.L.A.B.; Barreto, H.M.; Bandeira, P.N.; Nogueira, C.E.S.; Sena, D.M.; et al. Synthesis, Spectroscopic Characterization and Antibacterial Evaluation by Chalcones Derived of Acetophenone Isolated from Croton Anisodontus Müll.Arg. J. Mol. Struct. 2021, 1226, 129403. [CrossRef] 
30. Rani, A.; Anand, A.; Kumar, K.; Kumar, V. Recent Developments in Biological Aspects of Chalcones: The Odyssey Continues. Expert Opin. Drug Discov. 2019, 14, 249-288. [CrossRef]

31. Murti, Y.; Goswami, A.; Mishra, P. Synthesis and Antioxidant Activity of Some Chalcones and Flavanoids. Int. J. PharmTech Res. 2013, 5, 811-818.

32. Rocha, D.H.A.; Vaz, P.A.A.M.; Pinto, D.C.G.A.; Silva, A.M.S. Synthesis Chalones and Their Isomerization into Flavanones and Azaflavanones. MPs 2019, 2, 70. [CrossRef]

33. Liu, F.; Yang, J.-F.; Liu, H.; Wei, W.-Z.; Ma, Y.-M. Facile Microwave-Assisted Synthesis of 1,3,5-Trisubstituted Pyrazoline Derivatives Incorporating Sulfonyl Moiety. J. Chin. Chem. Soc. 2016, 63, 254-260. [CrossRef]

34. Narender, T.; Venkateswarlu, K.; Vishnu Nayak, B.; Sarkar, S. A New Chemical Access for 3'-Acetyl-4'-Hydroxychalcones Using Borontrifluoride-Etherate via a Regioselective Claisen-Schmidt Condensation and Its Application in the Synthesis of Chalcone Hybrids. Tetrahedron Lett. 2011, 52, 5794-5798. [CrossRef]

35. Narender, T.; Papi Reddy, K. A Simple and Highly Efficient Method for the Synthesis of Chalcones by Using BorontrifluorideEtherate. Tetrahedron Lett. 2007, 48, 3177-3180. [CrossRef]

36. Lahyani, A.; Chtourou, M.; Frikha, M.H.; Trabelsi, M. Amberlyst-15 and Amberlite-200C: Efficient Catalysts for Aldol and Cross-Aldol Condensation under Ultrasound Irradiation. Ultrason. Sonochem. 2013, 20, 1296-1301. [CrossRef]

37. Khaligh, N.G.; Mihankhah, T. Aldol Condensations of a Variety of Different Aldehydes and Ketones under Ultrasonic Irradiation Using Poly(N-Vinylimidazole) as a New Heterogeneous Base Catalyst under Solvent-Free Conditions in a Liquid-Solid System. Chin. J. Catal. 2013, 34, 2167-2173. [CrossRef]

38. Braun, R.U.; Ansorge, M.; Müller, T.J.J. Coupling-Isomerization Synthesis of Chalcones. Chem. Eur. J. 2006, 12, 9081-9094. [CrossRef] [PubMed]

39. Guo, T.; Jiang, Q.; Yu, L.; Yu, Z. Synthesis of Chalcones via Domino Dehydrochlorination/Pd(OAc)2-Catalyzed Heck Reaction. Chin. J. Catal. 2015, 36, 78-85. [CrossRef]

40. Eddarir, S.; Cotelle, N.; Bakkour, Y.; Rolando, C. An Efficient Synthesis of Chalcones Based on the Suzuki Reaction. Tetrahedron Lett. 2003, 44, 5359-5363. [CrossRef]

41. Xu, C.; Chen, G.; Huang, X. Chalcones by the Wittig Reaction of a Stable Ylide with Aldehydes Under Microwave Irradiation. Org. Prep. Proced. Int. 1995, 27, 559-561. [CrossRef]

42. Rioux, B.; Peyrot, C.; Mention, M.M.; Brunissen, F.; Allais, F. Sustainable Synthesis of P-Hydroxycinnamic Diacids through Proline-Mediated Knoevenagel Condensation in Ethanol: An Access to Potent Phenolic UV Filters and Radical Scavengers. Antioxidants 2020, 9, 331. [CrossRef]

43. Peyrot, C.; Mention, M.M.; Brunissen, F.; Balaguer, P.; Allais, F. Innovative Bio-Based Organic UV-A and Blue Light Filters from Meldrum's Acid. Molecules 2020, 25, 2178. [CrossRef] [PubMed]

44. Rozmer, Z.; Perjési, P. Naturally Occurring Chalcones and Their Biological Activities. Phytochem. Rev. 2016, 15, 87-120. [CrossRef]

45. Cui, W.; Zhang, J.; Wang, Q.; Gao, K.; Zhang, W.; Yang, J. A Novel Synthesis of Naringenin and Related Flavanones. J. Chem. Res. 2014, 38, 686-689. [CrossRef]

46. Al Bittar, S.; Mora, N.; Loonis, M.; Dangles, O. A Simple Synthesis of 3-Deoxyanthocyanidins and Their O-Glucosides. Tetrahedron 2016, 72, 4294-4302. [CrossRef]

47. Le Bail, J.-C.; Champavier, Y.; Chulia, A.-J.; Habrioux, G. Effects of Phytoestrogens on Aromatase, $3 \beta$ and $17 \beta$-Hydroxysteroid Dehydrogenase Activities and Human Breast Cancer Cells. Life Sci. 2000, 66, 1281-1291. [CrossRef]

48. Calliste, C.A.; Le Bail, J.C.; Trouillas, P.; Pouget, C.; Habrioux, G.; Chulia, A.J.; Duroux, J.L. Chalcones: Structural Requirements for Antioxidant, Estrogenic and Antiproliferative Activities. Anticancer Res. 2001, 21, 3949-3956.

49. Adams, J.H. Influence of Bulky Substituents on the Syntheses of 4-Hydroxy-3,5-Dialkylflavanoids. J. Org. Chem. 1967, 32, 3992-3998. [CrossRef]

50. Zhao, J.; Yuan, Y.; Meng, X.; Duan, L.; Zhou, R. Highly Efficient Liquid-Phase Hydrogenation of Naringin Using a Recyclable Pd/C Catalyst. Materials 2018, 12, 46. [CrossRef] [PubMed]

51. Kusumawati, I.; Indrayanto, G. Chapter 15-Natural Antioxidants in Cosmetics. In Studies in Natural Products Chemistry; Atta-ur-Rahman, Ed.; Elsevier: Amsterdam, The Netherlands, 2013; Volume 40, pp. 485-505.

52. Pillai, S.; Oresajo, C.; Hayward, J. Ultraviolet Radiation and Skin Aging: Roles of Reactive Oxygen Species, Inflammation and Protease Activation, and Strategies for Prevention of Inflammation-Induced Matrix Degradation-A Review. Int. J. Cosmet. Sci. 2005, 27, 17-34. [CrossRef] [PubMed]

53. Pop, A.; Berce, C.; Bolfă, P.; Nagy, A.-L.; Catoi, C.; Dumitrescu, I.-B.; Silaghi-Dumitrescu, L.; Loghin, F. Evaluation of the Possible Endocrine Disruptive Effect of Butylated Hydroxyanisole, Butylated Hydroxytoluene and Propyl Gallate in Immature Female Rats. Farmacia 2013, 61, 202-211.

54. Pop, A.; Kiss, B.; Loghin, F. Endocrine Disrupting Effects of Butylated Hydroxyanisole (BHA-E320). Clujul Med. 2013, 86, 16-20. [PubMed]

55. Reano, A.F.; Chérubin, J.; Peru, A.M.M.; Wang, Q.; Clément, T.; Domenek, S.; Allais, F. Structure-Activity Relationships and Structural Design Optimization of a Series of p-Hydroxycinnamic Acids-Based Bis- and Trisphenols as Novel Sustainable Antiradical/Antioxidant Additives. ACS Sustain. Chem. Eng. 2015, 3, 3486-3496. [CrossRef] 
56. Kozlowski, D.; Trouillas, P.; Calliste, C.; Marsal, P.; Lazzaroni, R.; Duroux, J.-L. Density Functional Theory Study of the Conformational, Electronic, and Antioxidant Properties of Natural Chalcones. J. Phys. Chem. A 2007, 111, 1138-1145. [CrossRef] [PubMed]

57. Mouterde, L.M.M.; Peru, A.A.M.; Mention, M.M.; Brunissen, F.; Allais, F. Sustainable Straightforward Synthesis and Evaluation of the Antioxidant and Antimicrobial Activity of Sinapine and Analogues. J. Agric. Food Chem. 2020, 68, 6998-7004. [CrossRef]

58. Neeley, E.; Fritch, G.; Fuller, A.; Wolfe, J.; Wright, J.; Flurkey, W. Variations in IC50 Values with Purity of Mushroom Tyrosinase. Int. J. Mol. Sci. 2009, 10, 3811-3823. [CrossRef] [PubMed]

59. Xu, B.; Yu, Y.; Wan, P.; Wan, C.; Cao, S. Synthesis and Antityrosinase, Antioxidant Activities of Phloretin Thiosemicarbazones. Res. Chem. Intermed. 2014, 40, 3095-3107. [CrossRef]

60. Ouchene, L.; Litvinov, I.V.; Netchiporouk, E. Hawaii and Other Jurisdictions Ban Oxybenzone or Octinoxate Sunscreens Based on the Confirmed Adverse Environmental Effects of Sunscreen Ingredients on Aquatic Environments. J. Cutan. Med. Surg. 2019, 23, 648-649. [CrossRef]

61. Boyd, A.; Stewart, C.B.; Philibert, D.A.; How, Z.T.; El-Din, M.G.; Tierney, K.B.; Blewett, T.A. A Burning Issue: The Effect of Organic Ultraviolet Filter Exposure on the Behaviour and Physiology of Daphnia Magna. Sci. Total Environ. 2021, 750, 141707. [CrossRef] [PubMed]

62. Siller, A.; Blaszak, S.C.; Lazar, M.; Olasz Harken, E. Update About the Effects of the Sunscreen Ingredients Oxybenzone and Octinoxate on Humans and the Environment. Plastic Surg. Nurs. 2018, 38, 158-161. [CrossRef] 